\title{
An Auxin-Mediated Regulatory Framework for Wound-Induced Adventitious Root Formation in Tomato Shoot Explants
}

Aurora Alaguero-Cordovilla, Instituto de Bioingeniería, Universidad Miguel Hernández, 03202 Elche, Spain, aalaguero@umh.es

Ana Belén Sánchez-García, Instituto de Bioingeniería, Universidad Miguel Hernández, 03202 Elche, Spain, ana.sanchezg@umh.es

Sergio Ibáñez, Instituto de Bioingeniería, Universidad Miguel Hernández, 03202 Elche, Spain, sibaez94@gmail.com

Alfonso Albacete, Instituto Murciano de Investigación y Desarrollo Agrario y Alimentario (IMIDA), 30150 La Alberca, Spain, alfonsoa.albacete@ carm.es

Antonio Cano, Departamento de Biología Vegetal, Universidad de Murcia, 30100 Murcia, Spain, aclario@um.es

Manuel Acosta, Departamento de Biología Vegetal, Universidad de Murcia, 30100 Murcia, Spain, macosta@um.es

José Manuel Pérez-Pérez, Instituto de Bioingeniería, Universidad Miguel Hernández, 03202 Elche, Spain, jmperez@umh.es

Corresponding author: José Manuel Pérez-Pérez (jmperez@umh.es)

Running Head: Wound-induced AR formation in 'Micro-Tom'

Word count breakdown: Abstract, 184; Introduction, 769; Materials and Methods, 1824; Results, 4000; Discussion, 2031; References, 2744; Fig. Legends, 1330.

Figures: 9

Supporting

Information: 5 


\section{ABSTRACT}

Adventitious roots (ARs) are produced from non-root tissues in response to different environmental signals, such as abiotic stresses, or after wounding, in a complex developmental process that requires hormonal crosstalk. Here, we characterized AR formation in young seedlings of Solanum lycopersicum cv. 'Micro-Tom' after whole root excision by means of physiological, genetic and molecular approaches. We found that a regulated basipetal auxin transport from the shoot and local auxin biosynthesis triggered by wounding are both required for the re-establishment of internal auxin gradients within the vasculature. This promotes cell proliferation at the distal cambium near the wound in well-defined positions of the basal hypocotyl and during a narrow developmental window. In addition, a pre-established pattern of differential auxin responses along the apical-basal axis of the hypocotyl and an as of yet unknown cell-autonomous inhibitory pathway contribute to the temporal and spatial patterning of the newly formed ARs on isolated hypocotyl explants. Our work provides an experimental outline for the dissection of wound-induced AR formation in tomato, a species that is suitable for molecular identification of gene regulatory networks via forward and reverse genetics approaches.

\section{KEYWORDS}

Solanum lycopersicum, cell reprogramming, auxin biosynthesis, polar auxin transport, de novo root formation, auxin response, hormone regulation, mechanical damage, adventitious rooting, tissue regeneration. 


\section{INTRODUCTION}

Adventitious roots (ARs) develop post-embryonically from non-root tissues, such as stems and leaves, usually in response to challenging environmental conditions; they may also be induced by mechanical damage or during in vitro tissue culture (Bellini et al., 2014; Druege et al., 2019; Gonin et al., 2019). During normal development though, many plant species develop ARs to perform specialized functions, such as increasing soil foraging and water absorption (Mhimdi and Pérez-Pérez, 2020). AR formation involves several developmental stages (de Klerk et al., 1999), and the key regulatory events occurring during the induction phase result in the molecular reprogramming of some vascular-associated cells (Lakehal and Bellini, 2019). In Arabidopsis leaf explants, wounding induces jasmonic acid (JA) production that indirectly upregulates auxin biosynthesis in the whole leaf (Chen et al., 2016; Zhang et al., 2019). Auxin is then actively transported to the wounded site where it promotes the fate change from regenerationcompetent cells to root founder cells in the vasculature through WUSCHELRELATED HOMEOBOX 11 (WOX11) expression (Liu et al., 2014). In turn, WOX11 (and the partially redundant WOX12) along with other auxin responsive factors, upregulate the expression of WOX5 and LATERAL ORGAN BOUNDARIES DOMAIN 16 (LBD16), which trigger the development of the AR primordia (Hu and Xu 2016).

The rooting of stem cuttings is a common vegetative propagation practice in many ornamental plants. Petunia (Petunia $\times$ hybrida) has been proposed as an adequate experimental system to analyze the relationship between plant hormones and excision-induced AR formation in stem cuttings (Druege et al., 2016; Druege and Franken, 2019). Upon excision, wounding triggers the early accumulation of JA and ethylene in the stem base, while subsequent accumulation of the active auxin, indole-3-acetic acid (IAA), in the basal end of the cutting is dependent on the pre-established polar auxin 
transport from the shoot (Ahkami et al., 2013; 2014; Druege et al., 2014; Lischweski et al., 2015). Additional studies in this species have contributed to the understanding of the effects of the nutritional status of whole cuttings on AR formation (Ahkami et al., 2009; Klopotek et al., 2016), as well as the specialized functions of specific nutrients, such as nitrogen (Zerche et al., 2016; Yang et al., 2019) and iron (Hilo et al., 2017). However, the lack of functional genomics tools complicates the identification of the molecular players involved in AR initiation in these and other non-model crops.

Among the Solanaceae, cultivated tomato (Solanum lycopersicum L.) has been used as a model species for research in genetics, fruit development and biotic and abiotic stress resistance (Rothan et al., 2016, 2019). Soil flooding induces AR formation in tomato hypocotyls via crosstalk between ethylene signaling and polar auxin transport regulation (Negi et al., 2010; Vidoz et al., 2010). The AR overproducing phenotype of the aerial roots (aer) tomato mutant seems strictly linked to an altered distribution of active auxin along the stem because of auxin transport deregulation, but the causal mutation is still unknown (Mignolli et al., 2017). The key role of polar auxin transport during adventitious rooting was established in Arabidopsis thaliana early through physiological studies (Ludwig-Müller et al., 2005), and was later confirmed by the study of mutants affected in efflux carrier proteins of the ATP-binding cassette (ABC) and PIN-FORMED (PIN) families (Della Rovere et al., 2013; Sukumar et al., 2013). In these and other studies, the wound-induced signal subsequently modulated auxin content by affecting the expression of auxin biosynthesis and polar auxin transport genes, thereby stimulating AR induction (Huang et al., 2020). However, a systematic analysis of the factors that regulate wound-induced AR formation in tomato is missing. Due to its small size and short life cycle, the 'Micro-Tom' cultivar has been proposed as a model for functional genomics in this species (Emmanuel and Levy, 2002). Since then, a wealth of genetic resources has been developed for this tomato cultivar (Kobayashi et al., 2014; Shikata et 
al., 2016). Therefore, we chose the 'Micro-Tom' cultivar to investigate the relative contribution of polar auxin transport from the shoot and of local auxin biosynthesis during wound-induced AR formation. Our results showed that wound-induced AR formation in 'Micro-Tom' hypocotyls occurred de novo through auxin-mediated activation of specialized cambial cells. We provide strong evidence that both regulated polar auxin transport from the shoot and local auxin biosynthesis near the wounded site cooperatively contribute to the build-up of endogenous auxin response gradients in a stereotyped pattern that, in turn, negatively regulates some Aux/IAA genes, such as ENTIRE (also known as SIIAA9). We also found that local auxin biosynthesis could partially overcome the requirement for shoot-derived auxin during AR formation; hence, a functional root system could be obtained from a hypocotyl explant. 


\section{MATERIALS AND METHODS}

\section{Plant material and growth conditions}

Seeds of cultivated tomato (Solanum lycopersicum L.) lines (Supplementary Table S1) were obtained from the C.M. Rick Tomato Genetics Resource Center (http://tgrc.ucdavis.edu/). The tomato cultivar 'Micro-Tom', the DR5::GUS line and the near-isogenic lines (NILs) used in this study have been described previously (Supplementary Table S1). Seeds were surface-sterilized in $2 \%(\mathrm{w} / \mathrm{v}) \mathrm{NaClO}$ for $10 \mathrm{~min}$ and rinsed thoroughly with sterile distilled water (four times). Seeds were then transferred to wet chambers at $28{ }^{\circ} \mathrm{C}$ in a dark growth cabinet for $96 \mathrm{~h}$. Germinated seedlings with primary roots $>4 \mathrm{~mm}$ ( 0 days after sowing) were transferred to $65 \times 120$ $\mathrm{mm}$ (diameter $\times$ height) glass jars containing $75 \mathrm{~mL}$ of sterile one-halfMurashige and Skoog basal salt medium (Duchefa Biochemie, The Netherlands), $20 \mathrm{~g} \mathrm{~L}^{-1}$ sucrose (Duchefa Biochemie), $2.5 \mathrm{~g} \mathrm{~L}^{-1}$ Gelrite (Duchefa Biochemie), $0.5 \mathrm{~g} \mathrm{~L}^{-1}$ 2-(N-morpholino) ethanesulfonic acid (Duchefa Biochemie), and $2 \mathrm{~mL} \mathrm{~L}^{-1}$ Gamborg B5 vitamin solution (Duchefa Biochemie), at $\mathrm{pH}$ 5.8. Glass jars were transferred to a growth cabinet during $16 \mathrm{~h}$ light (average photosynthetic photon flux density of $50 \mu \mathrm{mol} \mathrm{m}^{-2} \mathrm{~s}^{-1}$ ) at $26 \pm 1{ }^{\circ} \mathrm{C}$, and $8 \mathrm{~h}$ darkness at $23 \pm 1{ }^{\circ} \mathrm{C}$. The formation of ARs was then induced by removing with a sharp scalpel the whole root system $2-3 \mathrm{~mm}$ above the hypocotyl-root junction of young tomato seedlings at the 100-101 growth stages (fully expanded cotyledons and first leaf $\sim 0.5 \mathrm{~cm}$; Feller $e t$ al., 1995) [0 days after whole root excision; 0 dae]. The shoot explants were transferred to new glass jars with $75 \mathrm{~mL}$ of the standard growing medium (SGM) described above. Each jar contained six or seven seedlings of the same genotype and/or treatment. All experiments were performed in duplicate with a minimum of six biological replicates. 


\section{Macroscopic studies of wound-induced AR formation}

Shoot explants or hypocotyl explants (obtained by sectioning shoot explants just below the cotyledons) were incubated for 21 days in SGM glass jars supplemented with $0.25 \mu \mathrm{M}$ 1-naphthalene acetic acid (NAA, Duchefa Biochemie). In another experiment, a $10-\mu \mathrm{L} 0.5 \%$ (w/v) agarose drop with $0.25 \mu \mathrm{M}$ NAA was applied directly to the distal (apical) end of the hypocotyl explants. To evaluate the effect of the chemical inhibition of polar auxin transport, we performed three experiments using shoot explants (Feller's stages 100-101). In the first experiment, the explants were incubated for 21 days in SGM glass jars supplemented with either $40 \mu \mathrm{M}$ 2-naphthoxyacetic acid (2-NOA; Sigma-Aldrich, St. Louis, MO, USA; Lanková et al., 2010), or $40 \mu \mathrm{M}$ N-1-naphthylphthalamic acid (NPA; Sigma-Aldrich; Vidoz et al., 2010). In the second experiment, a lanolin ring containing mock, $40 \mu \mathrm{M} 2$ NOA or $40 \mu \mathrm{M}$ NPA was applied directly below the cotyledons of the explants. In the third experiment, a $10-\mu \mathrm{L} 0.5 \%$ (w/v) agarose drop with 40 $\mu \mathrm{M}$ NPA was applied directly to the cotyledons. For gravitropism studies, shoot explants or hypocotyl explants grown on SGM were transferred to the growth chamber and reoriented relative to gravity by $0^{\circ}$ or $180^{\circ}$ for measuring several AR traits, as described below. Hypocotyl explants were excised into two or three fragments. These explants were then transferred to vertically oriented glass jars with $75 \mathrm{~mL}$ of SGM supplemented with 0 (mock) or 0.01 $\mu \mathrm{M}$ NAA, as well as with $50 \mu \mathrm{M}$ yucasin DF (YDF) for auxin biosynthesis inhibition (Tsugafune et al., 2017).

In all these cases, ARs arising from the hypocotyl were visually scored and periodically annotated during 21 dae. AR emergence was estimated based on the day before the annotation of the first AR. Maximum AR length, hypocotyl length and maximum shoot length were measured at 21 dae, unless otherwise indicated; the length of the hypocotyl with emerged ARs was also measured. To study wound-induced AR formation in older tomato seedlings, 
explants from the hypocotyl, the first node or the apex were obtained from 16-day-old seedlings (Feller's stages 102-103).

\section{Phytohormone extraction and analysis}

Three biological replicates, each consisting of hypocotyl thin sections ( $\sim 5 \mathrm{~mm}$ long) of several shoot explants, were collected from apical and basal ends of the shoot explants at 0,1 and 3 dae. The shoot apical distal ends (including the meristem and the emerging leaves) and the cotyledons were also harvested at 3 dae. Phytohormones were extracted from frozen tissues and analyzed according to Großkinsky et al. (2014) and Villacorta-Martín et al. (2015). Auxin metabolites were identified according to their exact molecular masses and retention times as determined from total ion chromatograms generated by U-HPLC-HRMS (Accela-Exactive, ThermoFisher Scientific, Waltham, MA, USA) analysis.

\section{Light and laser confocal scanning microscopy}

Five-millimeter basal sections from shoot explants were obtained at different time points during adventitious rooting. Samples were fixed in a paraformaldehyde/Triton solution $(1.85 \%$ volume/volume formaldehyde, $45 \%$ ethanol, $5 \%$ acetic acid, and $1 \%$ Triton $\mathrm{X}-100$ ) for 3 days at $4{ }^{\circ} \mathrm{C}$. The fixed tissue was rinsed three times in $0.1 \mathrm{M}$ sodium phosphate buffer $(\mathrm{pH} 7.2)$ before dehydration in a graded ethanol series $(50 \%, 70 \%, 90 \%$, and $96 \%$ ethanol, 30 min each). Dehydrated samples were then embedded in Technovit 7100 resin (Heraeus Kulzer $\mathrm{GmbH}$, Germany) according to the manufacturer's instructions with slight modifications, as follows. Samples were immersed in the pre-infiltration solution (50\% resin and $50 \%$ ethanol) for $2.5 \mathrm{~h}$. The stem cutting samples remained for $4 \mathrm{~h}$ in the infiltration solution under a light vacuum at $25^{\circ} \mathrm{C}$ and polymerized for $20 \mathrm{~h}$ at $4{ }^{\circ} \mathrm{C}$. Thin sections 
of $7 \mu \mathrm{m}$ thickness were cut using a tungsten microtome knife (MICROM International $\mathrm{GmbH}$, Germany) on an HS $350 \mathrm{~S}$ rotary microtome (MICROM International $\mathrm{GmbH})$. Sections were stained either with $0.05 \%$ weight/volume (w/v) toluidine blue (Sigma-Aldrich) or $0.05 \% \mathrm{w} / \mathrm{v}$ ruthenium red (Sigma-Aldrich) in water and mounted in Eukitt (Chem-Lab NV, Belgium). Samples were observed using a bright-field Motic BA210 microscope (Motic Spain, Spain) and selected images were captured with a built-in Moticam 580INT documentation station (Motic Spain) and processed with GIMP 2.10.12 (GIMP Development Team, http://www.gimp.org). For GUS staining, whole hypocotyl sections from shoot explants at 0,1 and 3 dae were incubated at $37{ }^{\circ} \mathrm{C}$ for $24 \mathrm{~h}$ in multiwell plates in the presence of the GUS staining solution and processed as described by Bustillo-Avendaño et al. (2018).

For laser scanning confocal microscopy (LCSM), a razor blade was used to make cuts of $\sim 400 \mu \mathrm{m}$ thickness on a Petri dish containing ice-cold $0.1 \mathrm{M}$ sodium phosphate buffer ( $\mathrm{pH} 7.2$ ), and then the cuts were fixed with $4 \% \mathrm{w} / \mathrm{v}$ paraformaldehyde, which was dissolved in $0.1 \mathrm{M}$ sodium phosphate buffer ( $\mathrm{pH}$ 7.2). The fixed tissue was rinsed three times with $0.1 \mathrm{M}$ sodium phosphate buffer and immersed in ClearSee solution (Kurihara et al., 2015). Before LCSM visualization, cleared samples were stained with $0.5 \%$ w/v calcofluor white on ClearSee solution for $2 \mathrm{~h}$ and then rinsed three times with ClearSee solution. Excitation of calcofluor white was achieved by using the $405 \mathrm{~nm}$ wavelength diode, while the fluorescence emission was collected between 425 and $475 \mathrm{~nm}$. Images of the vascular bundles were taken at different regions of the Z-position of the sample and cambium cells were counted on each bundle. 


\section{Immunolocalization of IAA}

The basal region of shoot explants was manually sectioned with a razor blade and immediately fixed in freshly prepared 3\% 1-ethyl-3(dimethylaminopropyl)-carbodiimide hydrochloride (EDAC) (SigmaAldrich) in $1 \times$ PBS containing $0.1 \%$ Triton X-100 (PBS-T) for $1 \mathrm{~h}$ on ice. The sample sections were post-fixed for $1-2 \mathrm{~h}$ in $4 \%$ paraformaldehyde in PBS-T and washed three times in PBS-T for $5 \mathrm{~min}$. The sample sections were then dehydrated and rehydrated with 30\%, 50\%, 70\%, 100\%, 70\%, 50\%, and $30 \%$ methanol in PBS-T (5 min each step) and incubated with $2 \%$ cellulase Onozuka R-10 (Duchefa Biochemie) for $30 \mathrm{~min}$ at $25^{\circ} \mathrm{C}$. After washing as above, sections were incubated overnight at $4{ }^{\circ} \mathrm{C}$ with a $1: 100$ dilution of the anti-IAA-C-monoclonal antibody (A0855; Sigma-Aldrich) in PBS-T. The sections were then rinsed three times for $10 \mathrm{~min}$ with PBS-T. Samples were incubated with 1:200 dilution of Alexa Fluor 647-conjugated donkey antimouse IgG (ThermoFisher Scientific) antibody for $2 \mathrm{~h}$ at $25^{\circ} \mathrm{C}$. The prepared samples were observed with LCSM as described elsewhere (BustilloAvendaño et al., 2018).

\section{RNA isolation and first-strand cDNA synthesis}

Total RNA from $\sim 100 \mathrm{mg}$ of powdered tomato hypocotyls from 3-6 seedlings at 0,1 and 4 days after whole root excision was extracted in triplicate using the Spectrum Plant Total RNA Kit (Sigma-Aldrich). Contaminating genomic DNA was removed via incubation for $20 \mathrm{~min}$ at 37 ${ }^{\circ} \mathrm{C}$ with 4 units of DNase I (Thermo Fisher Scientific, Waltham, MA, USA). After DNase I inactivation at $70{ }^{\circ} \mathrm{C}$ for $15 \mathrm{~min}$, RNA was used directly for downstream applications. First strand cDNA was synthesized with $1 \mu \mathrm{g}$ of purified RNA using SuperScript IV Reverse Transcriptase (Thermo Fisher 
Scientific), along with Oligo(dT)18 primers. The resulting cDNA was diluted by adding $40 \mu \mathrm{L}$ of sterile distilled water, reaching a final volume of $60 \mu \mathrm{L}$.

\section{Gene expression analysis by real-time quantitative PCR}

Primers amplified 93-324 base pairs of the cDNA sequences (Supplementary Table S2). To avoid amplifying genomic DNA, forward and reverse primers were designed to bound to different exons and to hybridize across consecutive exons. For real-time quantitative PCR, $14 \mu \mathrm{L}$ reactions were prepared with $7 \mu \mathrm{L}$ of SsoAdvanced Universal SYBR Green Supermix (Bio-Rad, Hercules, CA; USA), $4 \mu \mathrm{M}$ of specific primer pairs ( $5 \mu \mathrm{M}$ each), $1 \mu \mathrm{L}$ of cDNA, and $2 \mu \mathrm{L}$ of DNase-free water. PCR amplifications were carried out in 96-well optical reaction plates on a Step One Plus Real-Time PCR System (Thermo Fisher Scientific). Three biological and two technical replicates were performed for each gene. The thermal cycling program started with a step of $10 \mathrm{~s}$ at $95^{\circ} \mathrm{C}$, followed by 40 cycles $\left(15 \mathrm{~s}\right.$ at $95^{\circ} \mathrm{C}$ and $60 \mathrm{~s}$ at $60{ }^{\circ} \mathrm{C}$ ), and the melt curve (from 60 to $95^{\circ} \mathrm{C}$, with increments of $0.3{ }^{\circ} \mathrm{C}$ every $5 \mathrm{~s})$. The dissociation kinetics of the amplified products confirmed their specificity. Primer validation was performed by the absolute quantification method (Lu et al., 2012) using a standard curve that comprised equal amounts from each cDNA sample. Gene expression analyses were carried out using the $2^{-\Delta \Delta \mathrm{Ct}}$ method (Livak and Schmittgen, 2001). The housekeeping SlACTIN2 (Solyc03g078400) gene was chosen to ensure reproducibility (Dekkers et al., 2012; Expósito-Rodríguez et al., 2008). For each gene, the mean fold-change values relative to the basal part of 'Micro-Tom' hypocotyls at 0 dae were used for graphical representation. 


\section{Statistical analyses}

Descriptive statistics (e.g., average, standard deviation [SD], median, maximum, and minimum) were calculated using StatGraphics Centurion XV software (StatPoint Technologies, Inc. Warrenton, VA, USA) and SPSS 21.0.0 (SPSS Inc., Chicago, IL, USA). Data outliers were identified based on the Grubbs' test and excluded for posterior analyses as described elsewhere (Grubbs, 1969). One-sample Kolmogorov-Smirnov tests were performed to analyze the goodness-of-fit between the distribution of the data and a theoretical normal distribution. We performed multiple testing analyses using the ANOVA F-test or Fisher's least significant difference (LSD) methods ( $p$ value $<0.01$, unless otherwise indicated). Non-parametric tests (Student $t$, Mann-Whitney or Kolmogorov-Smirnov) were used when necessary (i.e., AR emergence and AR number).

\section{RESULTS}

\section{Environmental dependence of wound-induced AR formation in tomato shoot explants}

When whole-root excised shoot explants of the 'Micro-Tom' cultivar were grown under different light regimes, ARs emerged significantly earlier (p-value $=0.000$ ) in continuous light than in the $16 / 8 \mathrm{~h}$ light/dark photoperiod or in continuous darkness, but rooting capacity at 14 dae was higher under standard 16/8 h light/dark conditions (Supplementary Fig. S1A). We found significant differences ( $\mathrm{p}$-value $=0.000$ ) in the rooting capacity of shoot explants at 10 days after whole-root excision (10 dae), with the highest values obtained in standard 16/8 h light/dark conditions (Supplementary Fig. S1B, C). We did not find significant differences ( $p$-value=0.123) in any of the studied parameters with regard to the gelling agent concentration 
(Supplementary Fig. S1D-F), and a slight decrease in AR emergence and rooting capacity at 14 dae without sucrose (Supplementary Fig. S1G-I). Hence, we selected 16/8 h light/dark, $2 \%$ sucrose and $0.25 \%$ w/v Gelrite as the standard conditions to study wound-induced AR formation in 'MicroTom' shoot explants.

\section{Cellular changes in the distal cambium anticipate the initiation of wound-induced Ars}

To understand the cellular events leading to wound-induced AR formation in 'Micro-Tom' shoot explants, we studied transverse sections of the basal region of the hypocotyl between 0 and 4 dae (Fig. 1). Before wounding ( 0 dae), the hypocotyl contained four vascular bundles, which were orthogonally arranged (Fig. 1A, 0 dae). Each vascular bundle was radially composed of external phloem tissue, several layers (3-5) of isodiametric cambial cells and internal xylem vessels (Fig. 1A, 0 dae). Despite the clear discontinuity of the xylem, phloem tissues and cambial cells were also observed between the vascular bundles (Fig. 1A, 0 dae). AR primordia within the hypocotyl were visualized by DR5::GUS marker expression (Welander $e t$ $a l ., 2014)$. We found one preformed AR primordia in the most basal region of the 'Micro-Tom' shoot explants before whole root excision, and this number significantly increased afterwards (Fig. 1B, C). Interestingly, the newly formed AR primordia were regularly spaced along the apical-basal axis of the hypocotyl within a narrow region and at a certain distance from the wounded tissue (Fig. 1C, 3 dae). The earliest morphological event related to AR initiation [i.e., initiation phase (de Klerk et al., 1999)] occurred at 2 dae, when small clusters of dividing cells located at the edge of the vascular bundles were observed (Fig. 1A, 2 dae). These cells seemed to originate from the cambial rows facing towards the phloem (i.e., distal cambium). Even at these early stages, we confirmed that these presumptive AR initiation foci 
expressed the auxin-responsive DR5::GUS marker at higher levels in the distal tip of the primordium (Fig. 1D, 2 dae), when some root-like internal anatomy was observed, such as the lateral root cap and the vascular initials (Fig. 1A, dotted section in 2 dae). These AR primordia eventually grew over the bundle sheath cells (Fig. 1D, 3 dae) and through the cortex [i.e., expression phase (de Klerk et al., 1999)], with an organized internal structure that fully resembled a mature root meristem, which later emerged through the epidermis (Fig. 1A, 4 dae). At this point, the internal vasculature of the ARs was connected to the xylem vessels of the hypocotyl bundles, and the continuity of the cambium was maintained through the pericycle cells of the emerging ARs (Fig. 1A, 4 dae).

\section{Auxin is required for $A R$ induction in the hypocotyl upon wounding}

Auxin, which is routinely used to induce ARs in stem cuttings when applied exogenously (Druege et al., 2019), is a well-known endogenous player of AR formation in several species (Pacurar et al., 2014), including the 'Micro-Tom' tomato cultivar (Guan et al., 2019). We detected endogenous IAA in some vascular cells near the xylem in the basal region of the explant at 2 dae by immunolocalization (Fig. 1E, white arrow). We measured the concentration of IAA in both the apical and basal regions of the shoot explants during AR formation by HPLC-MS (Fig. 1F). IAA levels were similarly partitioned between the apical- and basal-half of the hypocotyl before wounding (Fig. 1G, 0 dae). Between 1 and 2 dae, IAA levels were much higher in the basal-half of the hypocotyl than in its apical region (Fig. $1 \mathrm{G})$, which is indicative of the generation of an internal auxin gradient within the hypocotyl after whole root excision. Interestingly, at 4 dae, and consistent with the expression phase of AR formation (see above), the internal auxin levels were similar in the apical-derived and basal-derived regions of the hypocotyl (Fig. 1G). 
To confirm the functional relevance of the endogenous IAA gradient observed in the basal region of the hypocotyl at 1 dae, we studied the expression of two Aux/IAA genes, SIIAA11 and SIIAA12 (Supplementary Fig. S2A, D), which are known to be upregulated by auxin in tomato (AudranDelalande et al., 2012). SIIAAI1 and SIIAA12 genes were differentially upregulated in the basal region of the hypocotyl at 1 dae (p-value $=0.000$; Fig. $1 \mathrm{H}$ ), mirroring the high IAA levels (Fig. 1G) and DR5::GUS expression in this region at 1 dae (Fig. 1C). In Arabidopsis leaf explants, wounding indirectly upregulates several YUCCA $(Y U C)$ genes involved in IAA biosynthesis in the whole mesophyll, which then enhances rooting by increasing auxin levels near the wounding via polar auxin transport (Chen et al., 2016). Hence, we studied the expression of some auxin biosynthesis genes, such as SITAR2 $a$ and SIYUC2/6 (Supplementary Fig. S2B, C, E, F), in shoot explants during rooting, and we found a slight increase (p-value $=0.000$ ) in SIYUC2/6 expression at 1 dae as compared to their steady-state levels at 0 dae (Fig. 1I).

To determine the relevance of the endogenous auxin source for AR formation, we studied hypocotyl explants obtained by sectioning shoot explants just below the cotyledons. These hypocotyl explants lacked the IAA supply from the shoot apex and showed a significant delay ( $\mathrm{p}$-value=0.015) in AR emergence $(8.3 \pm 5.7$ dae $)$ in comparison with shoot explants with an intact auxin source (Fig. 2A). As a result, the rooting capacity at 14 dae was much lower ( $\mathrm{p}$-value $=0.000$ ) in hypocotyl explants than that in shoot explants (Fig. 2B). We observed that incubation of the explants with $0.25 \mu \mathrm{M}$ NAA $\left(\mathrm{NAA}_{\mathrm{m}}\right)$ induced abundant tissue over proliferation (i.e., callus-like growth) at the basal end of the explants, even in the absence of the shoot apex (Fig. $2 \mathrm{C}, \mathrm{D})$. Therefore, AR emergence was severely delayed ( $\sim 3$ dae) by NAA $m$ treatment in shoot and hypocotyl explants (Fig. 2A). The NAAm treatment significantly increased ( $\mathrm{p}$-value $=0.024$ ) rooting capacity in shoot explants at 14 dae by about 3-fold (Fig. 2B, C), but the emerging ARs were significantly 
shorter ( $p$-value=0.000; Fig. 2E). The basal region of the hypocotyl that produced ARs (hereinafter referred to as the AR formative region) enlarged upward by the NAA $\mathrm{N}_{\mathrm{m}}$ treatment in both cases (Fig. 2F). To discard local inhibitory effects caused by high NAA concentration at the basal end of the explants, we directly applied $0.25 \mu \mathrm{M}$ NAA on the distal end of the hypocotyl explants $\left(\mathrm{NAA}_{\mathrm{d}}\right)$, which might then be actively transported to the proximal region near the wound (Hošek et al., 2012). Accordingly, the AR emergence delay caused by the excision of the shoot apex was fully rescued by the noncell-autonomous $\mathrm{NAA}_{d}$ treatment (Fig. 2A) and their rooting capacity was also enhanced with respect to that of the hypocotyl explants without NAA (Fig. 2B, C).

\section{Auxin transport inhibition primarily affects wound-induced AR formation}

Our results suggest that auxin from the distal shoot is required for AR formation in the proximal (basal) hypocotyl region near the wound. To evaluate the role of basipetal auxin transport through the wounded hypocotyl, we applied 2-NOA or NPA, which respectively inhibit auxin influx (Lanková et al., 2010) or auxin efflux (Teale and Palme, 2018). AR emergence was completely blocked in shoot explants treated with NPA in the medium $\left(\mathrm{NPA}_{\mathrm{m}}\right)$ and there was a significant delay $(\mathrm{p}$-value $=0.000)$ in AR emergence in samples treated with $2-\mathrm{NOA}_{\mathrm{m}}(12.3 \pm 3.0$ dae $)$ with respect to the mocktreated samples $(4.8 \pm 0.7$ dae; Fig. $3 \mathrm{~A})$. Shoot explants treated with $2-\mathrm{NOA}$ $\left(2-\mathrm{NOA}_{\mathrm{m}}\right)$ displayed profuse tissue proliferation (i.e., callus-like growth; Fig. $3 \mathrm{~B}, \mathrm{C})$ in their basal region, with numerous $\mathrm{AR}$ primordia that were unable

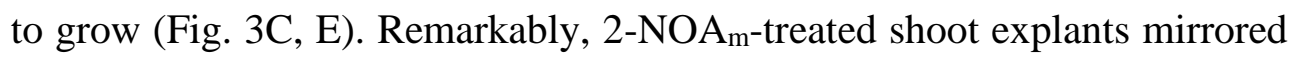
the NAA $(0.25 \mu \mathrm{M})$ effect observed previously, with the only difference being an increased AR number on the latter (Fig. 2C). Furthermore, there was 
no sign of tissue proliferation on the hypocotyl of $\mathrm{NPA}_{\mathrm{m}}$-treated shoot explants, and their shoot growth was also severely delayed (Fig. 3C).

To evaluate whether the local inhibition of auxin transport affected wound-induced AR formation, we applied a lanolin collar of 2-NOA (2$\mathrm{NOA}_{c}$ ) or NPA $\left(\mathrm{NPA}_{c}\right)$ just below the cotyledons. We found that $2-\mathrm{NOA}_{\mathrm{c}}$ or $\mathrm{NPA}_{c}$ produced a mild but statistically significant delay $(\mathrm{p}$-value $=0.013)$ in AR initiation as compared with the control treatment (Fig. 3A). Therefore, the rooting capacity was significantly reduced ( $\mathrm{p}$-value $=0.003$ ) with regard to the control treatment, and these differences were higher in the $\mathrm{NPA}_{\mathrm{c}}$-treated samples (Fig. 3D). In turn, the ARs produced from the NPA $\mathrm{A}_{\mathrm{c}}$-treated shoot explants were shorter than in mock $_{\mathrm{c}^{-}}$or $2-\mathrm{NOA}_{\mathrm{c}}$-treated ones at 21 dae (Fig. $3 E)$. Interestingly, the length of the $A R$ formative region was slightly increased in 2-NOA ${ }_{c}$, -treated shoot explants (Fig. 3F). We next evaluated wound-induced AR formation in the diageotropica mutant, which was defective on a cyclophilin protein putatively required for auxin efflux regulation (Oh et al., 2006; Ivanchenko et al., 2015). AR emergence was delayed in the diageotropica shoot explants with regard to its 'Micro-Tom' background, which also showed reduced rooting capacity at 14 dae (Fig. 3G), mirroring the mild effects caused by inhibiting polar auxin transport through the hypocotyl with local NPA application (NPAc; see above).

\section{Wound-induced AR initiation is independent of gravitropism}

Shoot or hypocotyl explants in vertically oriented jars or reoriented by $180^{\circ}$ relative to the gravity vector (Fig. 4A) showed non-significant differences $(p$-value $=0.748)$ in their wound-induced AR emergence (Fig. 4B) or in their rooting capacity at 14 dae (Fig. 4C). In addition, we did not find significant differences in AR lengths ( $p$-value=0.075) among the four conditions tested (Fig. 4D). In Arabidopsis, auxin accumulates at the lower side of hypocotyls during gravity stimulation, ultimately leading to 
asymmetric growth and organ bending (Su et al., 2017). We did not observe bending of 'Micro-Tom' hypocotyls (measured below the cotyledons) but we found bending against the gravity vector of the newly produced shoots (Fig. $4 \mathrm{E})$. The angle of emerging ARs relative to the hypocotyl was similar in the four experiments, although the growing roots later reoriented their growth towards the gravity vector (Fig. 4E). Intriguingly, we found that although vertically oriented shoot explants hardly produced additional ARs from 14 dae onwards, the shoot explants that were reoriented by $180^{\circ}$ produced $\sim 1.5$ fold more ARs at 21 dae. This increase in AR numbers in reoriented shoot explants might be caused by the AR formative region expanding upward (Fig. 4F).

\section{Endogenous IAA produced both in the shoot and the cotyledons are required for wound-induced AR emergence}

In stem cuttings of different species, the auxin produced in mature leaves accumulates in the stem region above the wound and triggers $A R$ formation within the vasculature (Druege et al., 2019). In 'Micro-Tom' shoot explants, both the shoot apex and the cotyledons might act as a direct source for the auxin required for wound-induced AR formation. Hence, we performed a series of experiments to address this hypothesis (Fig. 5A). Hypocotyl explants showed a significant delay ( $\mathrm{p}$-value $=0.000)$ in $\mathrm{AR}$ emergence (7.8 \pm 3.7 dae; Fig. $5 \mathrm{~B})$ as compared to that of shoot explants that included both the shoot apex and the cotyledons ( $4.6 \pm 0.5$ dae; Fig. $5 \mathrm{~B})$. We did not find significant differences in AR emergence between explants with deletions of either the shoot apex, or the shoot apex and one cotyledon, or both cotyledons but retaining the shoot apex (Fig. 5A-B), suggesting that the auxin signal required for wound-induced AR emergence was produced both in the cotyledons and in the shoot apex. 
We observed striking differences in the rooting capacity for the assayed conditions. On the one hand, we did not find significant differences ( $p$ value $=0.172$ ) in rooting capacity at 14 dae between shoot, 1-cotyledon, and 1-leaf explants (Fig. 5C). The 2-cotyledon explants (without the shoot apex) produced a significant increase ( $\mathrm{p}$-value $=0.004)$ in rooting capacity at 14 dae (Fig. 5C, D), with $\sim 1.5$ more ARs at 21 dae on average than whole shoot explants containing both cotyledons and the shoot apex. However, hypocotyl explants showed a significant decrease (p-value=0.000) in their rooting capacity $(1.7 \pm 0.6$ ARs at 14 dae; Fig. $5 \mathrm{C}, \mathrm{D})$. These results suggested that the cotyledons were the major source of auxin required for wound-induced AR emergence and that the shoot apex acted both as a source and a sink for auxin. To confirm this hypothesis, we locally applied the auxin efflux inhibitor NPA on cotyledons (referred to as shoot NPA $_{\text {cot }}$ explants), which resulted in a significant reduction ( $\mathrm{p}$-value $=0.000$ ) in the rooting capacity of the shoot explants at 14 dae $(2.0 \pm 0.6$ ARs at 14 dae; Fig. 5C, D), mirroring the results found on the hypocotyl explants (see above). We measured the endogenous levels of the active auxin (IAA) and of the inactive form of IAA conjugated to aspartic acid (IAA-Asp) in the shoot apex and the cotyledons of shoot explants at 3 dae, confirming a higher ratio of IAA to IAA-Asp in the cotyledons than in the shoot apex (Fig. 5E).

Intriguingly, AR emergence was not significantly altered ( $\mathrm{p}$ value $=0.471)$ in the shoot $\mathrm{NPA}_{\text {cot }}$ explants, suggesting that auxin from the shoot apex was sufficient to trigger AR initiation. Following the differences in AR emergence described earlier, the shortest AR length was observed in hypocotyl explants (Fig. 5F). In addition, the size of the AR formative region in the basal hypocotyl was a direct read-out of endogenous auxin levels, as this domain was significantly expanded (p-value $=0.00$ ) upwards in explants without the shoot apex (Fig. 5G). 


\section{A pre-established auxin response gradient within the hypocotyl supports wound-induced AR development}

Our results thus far suggested that auxin levels within the hypocotyl were dynamically established by basipetal auxin transport from the shoot apex and cotyledons. However, in the absence of this endogenous auxin source, the hypocotyl explants were able to develop a few ARs, which might have been non-cell autonomously induced by the residual auxin within the explant or by an unknown cell-autonomous signal. We dissected the hypocotyl explants in three fragments (apical, central, and basal) of similar lengths $(2.6 \pm 0.7 \mathrm{~mm}$; Fig. 6A). The central explants displayed an intermediate AR emergence response ( $6.8 \pm 5.8$ dae) compared to those of the apical-derived explants or of the basal-derived explants (Fig. 6B, solid lines). Consistent with these results, we found significant differences (pvalue $=0.000$ ) in the rooting capacity of the explants according to their initial position along the apical-basal axis of the hypocotyl, with the highest rooting capacity observed in the basal-derived explants ( $3.4 \pm 0.8$ ARs at 14 dae; Fig. 6C, D). Indeed, the differences found in AR lengths at 21 dae between the different explants (Fig. 6E) might be explained by the earliest AR emergence observed in the basal-derived explants. The size of the AR formative region was slightly expanded upwards ( $\mathrm{p}$-value $=0.056$ ) according to the explant position along the apical-basal axis (Fig. 6F). The observed differences in AR formation might be caused by differences in endogenous auxin levels or in auxin responses of the explants along the apical-basal axis. Next, we assayed the sensitivity of the different hypocotyl regions to the exogenously applied synthetic auxin NAA. Exogenously applied NAA induced callus-like growth in the proximal (basal) end of the explants at similar levels, regardless of their position within the intact hypocotyl (Fig. 6G). AR emergence was significantly enhanced ( $p$-value $=0.002$ ) by NAA only in the apical-derived 
explants (Fig. 6B, dotted lines). However, NAA application did not significantly increase the rooting capacity of the explants (Fig. 6C).

To discard the possibility that the observed differences in explants along the apical-basal axis were mirroring the circulating IAA levels at the time of excision, we studied AR formation in explants obtained from sectioning hypocotyls in two fragments of different lengths (Fig. 7A). AR emergence was significantly delayed ( $\mathrm{p}$-value $=0.000$ ) in all three apicalderived explants (11.6 \pm 6.2 dae; Fig. 7B, apical) as compared with those of the basal-derived explants $(2.0 \pm 0.9$ dae; Fig. 7B, basal), which occurred irrespective of their length in all cases ( $p$-value=0.982). Interestingly, incubation of apical-derived explants with NAA almost fully complemented their AR emergence delay (5.0 \pm 0.7 dae; Fig. 7B, apical), while it slightly delayed the AR emergence of the basal-derived explants ( $2.8 \pm 0.8$ dae; Fig. $7 \mathrm{~B}$, basal). However, rooting capacity at 14 dae was only significantly higher in the basal-derived explants ( $p$-value $=0.000)$ irrespective of their length ( $p$ value=0.117; Fig. 7C). Callus-like tissue formation at the proximal (basal) end of the explants in response to NAA incubation depended on their apicalbasal polarity with respect to the intact hypocotyls (see above). Indeed, only the apical-derived explants containing a large portion of the hypocotyl (C3_DBC) displayed higher levels of callus-like formation in response to exogenously applied NAA (p-value=0.000; Fig. 7D). Additionally, NAAtreatment increased callus-like formation in all basal-derived explants (i.e., containing the A region of the hypocotyl; Fig. 7D). These results support the hypothesis of an endogenous pre-established auxin response gradient within the hypocotyl before wounding, with higher auxin responses at the proximal (basal) end of the hypocotyl that determine wound-induced AR formation in response to endogenous auxin levels.

To assess whether auxin biosynthesis was locally activated by the wounding signal prior to AR formation in these explants, we studied the expression of several auxin biosynthesis genes in the apical (D, B) and basal 
(C, A) regions of the C2_DC and C2_BA explants (Fig. 7A). We found that SITAR $2 a$ and SITAR $2 b$ expression was significantly enhanced (pvalue $=0.000$ ) in the basal region of the explants at 2 dae (Fig. 7E). In addition, SIYUC2/6 expression was slightly induced at the basal region of the explants both before wounding and at 2 dae (Fig. 7E). We then evaluated the functionality of the endogenous auxin response gradient of the explants by studying Aux/IAA gene expression. SIIAA11 and SIIAA12 expression at 2 dae was significantly increased ( $\mathrm{p}$-value $=0.000$ ) in the basal region with respect to that of the basal region (Fig. 7F), mirroring the observed increase in auxin biosynthesis gene expression after wounding. Interestingly, while no expression gradient along the apical-basal axis was observed for these genes at 0 dae, a higher expression of SIIAAI1 and SIIAA12 was found in the basal $(\mathrm{C}, \mathrm{A})$ region of the explants with respect to that of the apical $(\mathrm{D}, \mathrm{B})$ region (Fig. 7F). These results suggested that, in 'Micro-Tom' hypocotyl explants, wounding acts locally to enhance auxin levels through the specific upregulation of SITAR2 genes in the tissues near the wound, and that the newly synthesized IAA is rapidly transported towards the most-basal region of the explants to activate specific target genes required for $\mathrm{AR}$ initiation. We then treated hypocotyl C2_DB and C2_BA explants with $50 \mu \mathrm{M}$ yucasin DF (YDF) that specifically inhibits YUC activity and hence IAA biosynthesis (Tsugafune et al., 2017). YDF did not affect AR emergence (Fig. 7G) but rooting capacity at 14 dae was significantly reduced (p-value=0.016) compared with the control treatment, mainly in the C2_BA explants (Fig. $7 \mathrm{H})$. In addition, wound-induced ARs were much shorter in YDF-treated explants at 21 dae (Fig. 7I). We also found that the size of the AR formative region was reduced by YDF (Fig. 7J), and confirmed that the YDF treatment specifically reduced AR formation (Fig 9A). Taken together, these results confirmed that, in 'Micro-Tom' hypocotyl explants, wounding regulates the formation of de novo auxin gradients near the basal region of the explants that are required for AR formation. 


\section{A functional auxin gradient in the basal region of the hypocotyl drives wound-induced $A R$ formation in tomato shoot explants}

To gain additional insight into the relevance of the endogenous auxin response to wound-induced AR formation in tomato shoot explants, we studied the entire mutant, which is defective in the Aux/IAA auxin repressor SIIAA9 (Zhang et al., 2007). We used a NIL of the entire mutant on the 'Micro-Tom' background with the characteristic simple leaf phenotype for our studies (Fig. 8A). AR emergence in the entire mutant was not significantly different ( $\mathrm{p}$-value $=0.065$ ) from that of the 'Micro-Tom' genetic background (Fig. 8B). However, the rooting capacity at 14 dae was significantly higher ( $\mathrm{p}$-value $=0.000$ ) in the entire mutant compared to that of the wild type (Fig. 8C), with a net difference of approximately 4 more ARs in entire at 21 dae than in 'Micro-Tom' (Fig. 8B), which nevertheless were of similar lengths in both genotypes (Fig. 8D). Interestingly, the AR formative region in the entire mutant was significantly expanded upward ( $\mathrm{p}$ value $=0.000$ ) as compared to that of the 'Micro-Tom' background (Fig. 8E and 9B). Different from that found for the C2_DC and C2_BA hypocotyl explants (see the previous section), we only observed minor changes in auxin biosynthesis gene expression along the apical-basal axis of 'Micro-Tom' shoot explants between 0 and 1 dae (Fig. 8F). However, we found that the entire mutant presented higher expression of the auxin biosynthesis genes SITAR $2 b$ and SIYUC2/6 in the basal region of the shoot explants at 1 dae when compared to 'Micro-Tom' (Fig. 8F), suggesting upregulation of auxin biosynthesis genes in the entire shoot explants after wounding. Besides, the rooting capacities at 14 dae of entire hypocotyl explants of different regions $\left(C 2 \_D C, C 2 \_B A\right)$ were slightly higher $(p$-value $=0.032)$ than those of the wild type (Fig. 8G). 
In the entire hypocotyls, the ARs originated from the same vascular region as those in the 'Micro-Tom' background (Fig. 8H, I). However, we observed a significant increase ( $p$-value $=0.000$ ) in the number of rows of cambial cells in the entire mutant at 1 dae (Fig. 8J), which, together with the enhanced auxin biosynthesis after wounding, would contribute to the higher rooting capacities observed in the shoot explants and hypocotyl explants of the entire mutant.

We wondered whether the wound-induced AR formation observed in the hypocotyl could also be elicited on higher nodes of young tomato seedlings. Hence, we studied wound-induced AR formation in three different regions of 16-day-old tomato seedlings (Fig. 9C). A high proportion ( 65\%) of explants from the first node did not produce any ARs even four weeks after excision, and the explants that had produced ARs were severely delayed in their initiation (Fig. 9C). Therefore, the rooting capacity of the explants from the first node was reduced (Fig. 9D), and their ARs were also shorter (Fig. 9E). We observed profuse tissue proliferation in the basal region of the explants from the first node that did not produce ARs (Fig. 9F, G), resembling hypocotyl explants treated with NAA (Fig. 2C). However, the explants from the shoot apex were mostly unable to produce any response in the basal region near the wound (Fig. 9C). These results confirmed that a developmental gradient along the apical-basal axis of the plant restricts wound-induced AR formation to the most basal tissues in 'Micro-Tom' seedlings.

\section{Variation of wound-induced AR formation in several tomato cultivars}

We previously studied several traits of the AR system in whole-root excised shoot explants in different tomato cultivars (Alaguero-Cordovilla et $a l ., 2018)$. AR emergence was significantly delayed (p-value $=0.000)$ in the 'Micro-Tom' cultivar when compared to that of the other studied cultivars (Supplementary Fig. S3A). Consequently, the rooting capacity was 
significantly reduced (p-value $=0.000)$ in the 'Micro-Tom' cultivar $(4.0 \pm 1.3$ ARs at 14 dae) as compared to those in 'Moneymaker', 'UC-82' or 'Heinz 1706-BG' (7.7 \pm 2.8 ; Supplementary Fig. S3B). Interestingly, AR formation was restricted to the most basal region of the hypocotyl in 'UC-82' while it was expanded upward in 'Moneymaker' and 'Heinz 1706-BG' (Supplementary Fig. S3C, D), irrespective of their hypocotyl lengths (Supplementary Fig. S3E). Furthermore, despite the differences in AR emergence between the studied cultivars, the length of the AR system at the end of the experiment (18 dae) did not significantly change ( $p$-value $=0.462$; Supplementary Fig. S3F). These results suggested that genetic variation within the tomato cultivars might contribute to variation in wound-induced AR system attributes and hence deserves further investigation.

\section{DISCUSSION}

AR formation is a critical developmental process in cutting propagation within the horticultural industry (Druege et al., 2019). Despite the key role of auxin in this process (Pacurar et al., 2014), our knowledge about the molecular determinants is still incomplete. In Arabidopsis hypocotyls, ARs are initiated from xylem pole pericycle cells (Sukumar et al., 2013) in a process resembling lateral root initiation (Bellini et al., 2014). However, ARs in adult tissues, such as excised leaves, originate from the proliferating lineage of some vascular-associated cells that express the WOX11 transcription factor (Liu et al., 2014; Hu and Xu 2016; Bustillo-Avendaño et al., 2018). In contrast to Solanum dulcamara, where preformed AR primordia in the stem emerge in response to flooding (Dawood et al., 2014), we found that wound-induced AR primordia in young 'Micro-Tom' hypocotyls were formed de novo after whole root excision (Fig. 9H). In our experimental system, AR initiation is temporally and spatially regulated by the stereotyped divisions of the distal cambium located at the edge of the vascular bundles. 
In the earliest stage, a small cluster of dividing cambial cells expresses the auxin-responsive DR5::GUS marker (stage 1; Bustillo-Avendaño et al., 2018), and in response, the root founder cells (RFCs) are then specified within this cluster (stage 2). In a recent report, Guan et al. (2019) localized the expression of the auxin reporter DR5pro:YFP in a subset of cambial cells during wound-induced AR formation in 'Micro-Tom' shoot explants, which is consistent with our findings with regards to the exact site of AR initiation. The lack of suitable cell-autonomous markers of cambial stem cell identity (Smetana et al., 2019) make detailed lineage analyses during AR formation in 'Micro-Tom' difficult. It has been reported that in petunia stem cuttings, several clusters of meristematic cells develop simultaneously within the same cambial ring in response to the auxin signal (Ahkami et al., 2014). In our study, however, consecutive ARs appeared to originate from opposite vascular rings and at a certain distance within the hypocotyl. This intriguing observation might be related to the internal vascular arrangement of the 'Micro-Tom' hypocotyl (vascular bundles arranged in a cross shape instead of the continuous vascular ring observed in petunia stem cuttings) or to an unknown lateral inhibition mechanism that spatially restricts AR initiation in the 'Micro-Tom' hypocotyl.

Day length was among the environmental factors that positively regulated AR initiation in 'Micro-Tom' hypocotyls, and sucrose exerted an additive effect on the rooting capacity of tomato shoot explants (this work). A previous study on tomato shoot explants found that the effect of light on AR formation was not directly related to photoreceptor-dependent light perception (Tyburski and Tretyn, 2004). Hence, the regulatory effect of light on adventitious rooting might result from its interaction with phytohormones, particularly auxin (Sorin et al., 2005; Gutierrez et al., 2009). Alternatively, light may influence AR formation due to its significant role in the synthesis of sugars. Studies on petunia cuttings highlighted the contribution of carbon allocation to AR formation sites in the basal region of the stem under different 
light regimes (Klopotek et al., 2016). Additional experiments using the 'Micro-Tom' model will enhance our understanding of the crosstalk between light, carbohydrate levels, and wound-induced AR formation.

In the current model for AR formation in stem cuttings (Druege et al., 2019), a pre-established polar auxin transport from mature leaves produced IAA accumulation in the basal region of the stem just above the wounding site, which triggered the de-differentiation and cell cycle reactivation of the neighboring cambial cells prior to AR initiation. We found that woundinduced AR formation in 'Micro-Tom' shoot explants resembled that of AR formation in stem cuttings. We observed the buildup of an internal IAA response gradient within the tomato hypocotyl shortly after whole root excision that reached its maximum levels at a defined position of the cambium, on a narrow proximal (basal) region of the hypocotyl just above the wound (Fig. 9I). In 'Micro-Tom' shoot explants, auxin is mainly produced in the cotyledons, and the emerging leaves are both a source and a sink for auxin (Fig. 9H). We found a direct correlation between the amount of auxinproducing tissues and the size of the AR-formative domain, which directly contributed to the rooting capacity of the explants (Fig. 9J). Indeed, local application of IAA in the proximal (basal) region of the hypocotyl resulted in the AR formative region significantly expanded upwards and concomitantly increased the number of wound-induced ARs (this work).

Using a combination of physiological and genetic approaches, we provided additional evidence of the key role of basipetal auxin transport in the regulation of wound-induced AR formation in 'Micro-Tom' shoot explants (Guan et al., 2019). First, we demonstrated that actively transported and shoot-derived IAA was required to trigger AR formation in the proximal (basal) hypocotyl region as the AR reduction caused by shoot decapitation could be fully rescued via local auxin application through the apical (distal) region of the explants. Disruption of basipetal auxin transport with 2-NOA and NPA also reduced the initiation of ARs in the basal region of the 
hypocotyl. In addition, the observation that local inhibition of auxin efflux in the basal hypocotyl domain blocked AR initiation suggested that transverse auxin flow, likely from the internal vasculature to the distal cambial cells, is also required for the formative divisions during AR initiation (Fig. 9I). Interestingly, the chemical inhibition of the auxin influx carriers AUX1/LAX by 2-NOA allowed the formation of callus-like tissue at the basal hypocotyl (resembling NAA-treated hypocotyl explants), which were severely delayed in AR formation. Indeed, in Arabidopsis hypocotyls, the activity of AUX1 is essential for AR initiation (da Costa et al., 2020), while other LAX members (such as LAX3) are required for AR emergence (Della Rovere et al., 2013; 2015). Consequently, when the new ARs emerged during the expression phase (de Klerk et al., 1999), they acted as active sinks for the basipetal auxin transported from the shoot, limiting the auxin overflow effect observed in the most basal region of the hypocotyl at earlier time-points. The tomato diageotropica mutant lacks lateral roots due to abnormal polar auxin transport that prevents the buildup of the auxin maxima in the vascular pericycle cells (Ivanchenko et al., 2015). DIAGEOTROPICA encodes a cyclophyllin protein with peptidyl-prolyl isomerase activity (Oh et al., 2006) that has been proposed to regulate PIN-mediated IAA efflux (Ivanchenko et al., 2015). As expected from a defect in basipetal auxin transport, we observed a reduction in the rooting capacity in wound-induced shoot explants of diageotropica mutants, a phenotype that mirrors our results on the chemical inhibition of polar auxin transport through the hypocotyl in 'MicroTom' seedlings. We found that gravistimulation enhanced the AR formative region, which might be consistent with a reduced flow of basipetal auxin transport through the hypocotyl, likely though PIN3 repolarization upon gravistimulation, as it occurs in Arabidopsis (Rakusová et al., 2011; 2016). Together, these results indicate that tightly regulated polar auxin transport through the hypocotyl is required to set up the AR formative region during wound-induced AR formation. 
We previously determined that the stabilization of the Aux/IAA repressor SOLITARY ROOT (SLR), also named IAA14, in Arabidopsis leaf explants reduced de novo root initiation downstream of the IAA28 module that regulates RFC specification (Bustillo-Avendano et al., 2018). The tomato ENTIRE gene encodes SIIAA9, a close SLR homolog (Wu et al., 2012), and the entire loss-of-function mutant displayed simple leaves instead of compound leaves but normal lateral root development (Zhang et al., 2007). We found that reduced levels of the SIIAA9 repressor enhanced woundinduced AR formation due to the apical expansion of the AR-formative domain. In addition, we found upregulation of two auxin biosynthesis genes in the basal region of the entire shoot explants after whole-root excision, which might directly contribute to the high endogenous auxin pool in this mutant. Our results suggest that reduced Aux/IAA levels in entire shoot explants lead to enhanced auxin responses that, in turn, lower the threshold for AR initiation at more distally located vascular bundles. Alternatively, the entire mutation might cause subtle developmental defects in the hypocotyl, which might alter the cellular interactions required for AR initiation. Downstream of the IAA14 repressor, the AUXIN RESPONSIVE FACTOR7 (ARF7) and ARF19 are required to activate formative divisions of pericycle cells during AR formation in etiolated Arabidopsis hypocotyls upon transfer to light (Lee et al., 2019). Several tomato homologs of ARF7 and ARF9 genes are expressed during the early stages of fruit development and their inactivation using RNAi renders fruits with thick pericarp due to increased cell expansion (de Jong et al., 2015; 2008). Additional experiments are ongoing to determine whether ARF7 and ARF9 inactivation affects woundinduced AR formation in tomato shoot explants. Since the tomato genome contains 25 Aux/IAA genes (Audran-Delalande et al., 2012) and 22 ARF genes (Zouine et al., 2014), multiple Aux/IAA and ARF genes might contribute to wound-induced AR formation and additional studies will be required to elucidate the Aux/IAA-ARF gene regulatory pathway(s) involved 
in this process. During wound-induced AR formation in Arabidopsis leaf explants, two parallel pathways downstream of the auxin signal converge on the upregulation of several genes of the LATERAL ORGAN BOUNDARIES DOMAIN (LBD) gene family, such as LBD16, LBD18, and LBD29 (Liu et al., 2014; Lee et al., 2019). Indeed, we found that two tomato genes encoding LBD16 and LBD29 homologs (Solyc09g066260 and Solyc09g066270) were differentially upregulated in stages 2 and 3 of wound-induced AR formation in 'Micro-Tom' hypocotyl explants suggesting a conserved developmental framework for de novo root formation in both species (E. Larriba and J.M. Pérez-Pérez, unpublished).

Our results with 'Micro-Tom' hypocotyl explants of different lengths provide a conceptual framework for the study of de novo organ formation in this species, as these explants were able to develop both ARs and adventitious shoots. We found striking differences in AR emergence and rooting capacity with respect to the position of the explants along the apical-basal axis of the hypocotyl, irrespective of the length of the explants used. Hence, an endogenous pre-pattern of auxin responses within the hypocotyl is already established during early development, which limits AR initiation to most basal tissues. Interestingly, the size of the AR formative region remained constant in all cases, suggesting their tight regulation. Our results also suggested that wound-induced AR formation in excised hypocotyl explants was independent of shoot-derived auxin but dependent on a short-range signal produced near the wounded tissue. In Arabidopsis, root meristem regeneration requires multiple auxin biosynthetic sources that are newly specified near the cut site and it has been suggested that regeneration competence relies on the ability to specify new local auxin sources in a precise temporal pattern (Matosevich et al., 2020). We found mild upregulation of some auxin biosynthesis genes in the excised hypocotyl explants upon wounding, while the auxin responses were subsequently localized to the most basal region of the explants, suggesting that polar auxin 
transport might contribute to the buildup of new auxin maxima in the basal region triggering AR initiation. In our working model for wound-induced $A R$ formation in excised hypocotyl explants, AR initiation is dependent on the extent of local auxin biosynthesis in the wounded tissue and the endogenous auxin response of the explants (Fig. 9J). We demonstrated that both factors varied according to the apical-basal position of the explant on the intact hypocotyl.

During wound-induced AR formation in Arabidopsis leaf explants, wound-induced JA directly induces the expression of ETHYLENE RESPONSE FACTOR109 (ERF109), which functions directly upstream of a key gene in the auxin biosynthesis pathway (Zhang et al., 2019; Ye et al., 2020). In addition, ERF109 activates ERF115 expression (Zhou et al., 2019), which in turn promotes stem cell renewal and grants regeneration competence after physical damage (Heyman et al., 2016). Two recent reports provide additional evidence of a mechanistic model for wound-induced stem cell regeneration that involves the sensing of damaged cells and the activation of local auxin signaling to coordinate downstream transcriptional responses near the wound through ERF115 activity (Canher et al., 2020; Hoermayer et al., 2020). The tomato genome contains several ERF109 and ERF115 homologs, some of which are differentially upregulated after wounding (E. Larriba and J.M. Pérez-Pérez, unpublished); hence, a similar regulatory network might be involved in the fine-tuning of wound-induced AR formation in excised tomato explants. 


\section{ACKNOWLEDGEMENTS}

We are grateful to María José Níguez-Gómez for her expert technical assistance. We thank Prof. Lázaro E. Peres (Universidade de São Paulo, Brazil) and Prof. Yoshinori Kanayama (Tohoku University, Japan) for providing lines in the 'Micro-Tom' background studied in this work, and Prof. Ken-Ichiro Hayashi (Okayama University of Science, Japan) for providing the yucasin DF auxin biosynthesis inhibitor. We thank several members of the Instituto de Bioingeniería (Universidad Miguel Hernández, Spain) for sharing their equipment. This work was supported by the Ministerio de Ciencia e Innovación of Spain (BIO2015-64255-R and RTI2018-096505-B-I00), the Conselleria d'Educació, Cultura i Esport of the Generalitat Valenciana (IDIFEDER 2018/016 and PROMETEO/2019/117), and the European Regional Development Fund (ERDF) of the European Commission. SI was a research fellow of the Generalitat Valenciana (ACIF/2018/220).

\section{CONFLICT OF INTEREST}

The authors declare that they have no competing financial interests as defined by Plant, Cell and Environment, or other interests that might be perceived to influence the results and/or discussion reported in this article.

\section{AUTHOR CONTRIBUTIONS}

Conceptualization and Supervision: J.M.P.-P.; Methodology, J.M.P.-P., A.A.-C., A.B.S.-C., S.I., and A.A.; Investigation, A.A.-C., A.B.S.-C., S.I., and A.C.; Formal Analysis: A.A.-C., A.C., and S.I.; Writing - Original Draft Preparation, J.M.P.-P., and A.A.-C.; Writing - Review \& Editing, J.M.P.-P., 
A.A., and M.A.; Funding Acquisition, J.M.P.-P.; Resources, J.M.P.-P., A.A., and M.A.

\section{DATA AVAILABILITY STATEMENT}

The datasets generated during and/or analysed during the current study are available from the corresponding author on reasonable request. 


\section{REFERENCES}

Acosta, M., Oliveros-Valenzuela, M. R., Nicolás, C., \& Sánchez-Bravo, J. (2009).

Rooting of carnation cuttings. Plant Signaling \& Behavior, 4, 234-236.

Aguinis, H., Gottfredson, R. K., \& Joo, H. (2013). Best-practice recommendations for defining, identifying, and handling outliers. Organizational Research Methods, 16, 270-301.

Agulló-Antón, M. Á., Ferrández-Ayela, A., Fernández-García, N., Nicolás, C., Albacete, A., Pérez-Alfocea, F., Sánchez-Bravo, J., Pérez-Pérez, J. M., \& Acosta M. (2014). Early steps of adventitious rooting: morphology, hormonal profiling and carbohydrate turnover in carnation stem cuttings. Physiologia Plantarum, 150, 446-462.

Ahkami, A., Scholz, U., Steuernagel, B., Strickert, M., Haensch, K-T., Druege, U., Reinhardt, D., Nouri, E., von Wirén, N., Franken, P., \& Hajirezaei M. R. (2014). Comprehensive transcriptome analysis unravels the existence of crucial genes regulating primary metabolism during adventitious root formation in Petunia hybrida. PLoS ONE, 9, e100997.

Ahkami, A. H., Lischewski, S., Haensch, K. T., Porfirova, S., Hofmann, J., Rolletschek, H., Melzer, M., Franken, P., Hause, B., Druege, U., \& Hajirezaei, M. R. (2009). Molecular physiology of adventitious root formation in Petunia hybrida cuttings: Involvement of wound response and primary metabolism. New Phytologist, 181, 613-625.

Alaguero-Cordovilla, A., Gran-Gómez, F., Tormos-Moltó, S., \& Pérez-Pérez, J. M. (2018). Morphological characterization of root system architecture in diverse tomato genotypes during early growth. International Journal of Molecular Sciences, 19, 3888.

Audran-Delalande, C., Bassa, C., Mila, I., Regad, F., Zouine, M., \& Bouzayen, M. (2012). Genome-wide identification, functional analysis and expression profiling of the Aux/IAA gene family in tomato. Plant and Cell Physiology, 53, 659-672. 
Bellini, C., Pacurar, D.I., \& Perrone, I. (2014). Adventitious roots and lateral roots: similarities and differences. Annual Review of Plant Biology, 65, 639-666.

Bustillo-Avendaño, E., Ibáñez, S., Sanz, O., Barros, J. A. S., Gude, I., PerianezRodriguez, J., Micol, J. L., del Pozo, J. C., Moreno-Risueno, M. A., \& PérezPérez, J. M. (2018). Regulation of hormonal control, cell reprogramming, and patterning during de novo root organogenesis. Plant Physiology, 176, 17091727.

Canher, B., Heyman, J., Savina, M., Devendran, A., Eekhout, T., Vercauteren, I., Prinsen, E., Matosevich, R., Xu, J., Mironova, V., \& De Veylder, L. (2020). Rocks in the auxin stream: Wound-induced auxin accumulation and ERF115 expression synergistically drive stem cell regeneration. Proceedings of the National Academy of Sciences, 117, 16667-16677.

Cano, A., Sánchez-García, A. B., Albacete, A., González-Bayón, R., Justamante, M. S., Ibáñez, S., Acosta, M., \& Pérez-Pérez, J. M. (2018). Enhanced conjugation of auxin by $\mathrm{GH} 3$ enzymes leads to poor adventitious rooting in carnation stem cuttings. Frontiers in Plant Science, 9, 566.

Chen, L., Tong, J., Xiao, L., Ruan, Y., Liu, J., Zeng, M., Huang, H., Wang, J. W., $\& \mathrm{Xu}, \mathrm{L}$. (2016). YUCCA -mediated auxin biogenesis is required for cell fate transition occurring during de novo root organogenesis in Arabidopsis. Journal of Experimental Botany, 67, 4273-4284.

da Costa, C. T., Offringa, R., \& Fett-Neto, A. G. (2020). The role of auxin transporters and receptors in adventitious rooting of Arabidopsis thaliana preetiolated flooded seedlings. Plant Science, 290, 110294.

Dawood, T., Rieu, I., Wolters-Arts, M., Derksen, E.B., Mariani, C., \& Visser, E. J. W. (2014). Rapid flooding-induced adventitious root development from preformed primordia in Solanum dulcamara. AoB Plants, 6, plt058. de Klerk, G. J., van der Krieken, W., \& de Jong, J. C. (1999). Review the formation of adventitious roots: New concepts, new possibilities. In Vitro Cellular \& Developmental Biology - Plant, 35, 189-199. 
Dekkers, B. J. W., Willems, L., Bassel, G. W., van Bolderen-Veldkamp, R. P. M., Ligterink, W., Hilhorst, H. W. M, \& Bentsink, L. (2012). Identification of reference genes for RT-qPCR expression analysis in Arabidopsis and tomato seeds. Plant \& Cell Physiology, 53, 28-37.

Della Rovere, F., Fattorini, L., D’Angeli, S., Veloccia, A., Del Duca, S., Cai, G., Falasca, G., \& Altamura, M. M. (2015). Arabidopsis SHR and SCR transcription factors and AUX1 auxin influx carrier control the switch between adventitious rooting and xylogenesis in planta and in in vitro cultured thin cell layers. Annals of Botany, 115, 617-628.

Della Rovere, F., Fattorini, L., D’Angeli, S., Veloccia, A., Falasca, G., \& Altamura, M. M. (2013). Auxin and cytokinin control formation of the quiescent centre in the adventitious root apex of Arabidopsis. Annals of Botany, 112, 1395-1407.

Díaz-Sala, C., Garrido, G., \& Sabater, B. (2002). Age-related loss of rooting capability in Arabidopsis thaliana and its reversal by peptides containing the Arg-Gly-Asp (RGD) motif. Physiologia Plantarum, 114, 601-607.

Druege, U., Franken, P., \& Hajirezaei, M. R. (2016). Plant hormone homeostasis, signaling and function during adventitious root formation in cuttings. Frontiers in Plant Science, 7, 381.

Druege, U., Franken, P., Lischewski, S., Ahkami, A.H., Zerche, S., Hause, B., \& Hajirezaei, M. R. (2014). Transcriptomic analysis reveals ethylene as stimulator and auxin as regulator of adventitious root formation in petunia cuttings. Frontiers in Plant Science, 5, 494.

Druege, U., \& Franken, P. (2019). Petunia as model for elucidating adventitious root formation and mycorrhizal symbiosis: at the nexus of physiology, genetics, microbiology and horticulture. Physiologia Plantarum, 165, 58-72.

Druege, U., Hilo, A., Pérez-Pérez, J.M., Klopotek, Y., Acosta, M., Shahinnia, F., Zerche, S., Franken, P., \& Hajirezaei, M. R. (2019). Molecular and physiological control of adventitious rooting in cuttings: phytohormone action meets resource allocation. Annals of Botany, 123, 929-949. 
Emmanuel, E., \& Levy, A. A. (2002). Tomato mutants as tools for functional genomics. Current Opinion in Plant Biology, 5, 112-117.

Expósito-Rodríguez, M., Borges, A. A., Borges-Pérez, A., \& Pérez, J. A. (2008). Selection of internal control genes for quantitative real-time RT-PCR studies during tomato development process. BMC Plant Biology, 8, 131.

Feller, C., Bleiholder, H., Buhr, L., Hack, H., Hess, M., Klose, R., Meier, U., Stauss, R., Van den Boom, T, \& Weber, E. (1995). Phänologische entwicklungsstadien von gemüsepflanzen: II. Fruchtgemüse und hülsenfrüchte. Nachrichtenblatt des Deutschen Pflanzenschutzdienstes, 47, 217-232.

Fukaki, H., Tameda, S., Masuda, H., \& Tasaka, M. (2002). Lateral root formation is blocked by a gain-of-function mutation in the SOLITARY-ROOT/IAAI4 gene of Arabidopsis. The Plant Journal, 29, 153-168.

Gonin, M., Bergougnoux, V., Nguyen, T. D., Gantet, P., \& Champion, A. (2019). What makes adventitious roots? Plants, 8, 240.

Großkinsky, D. K., Albacete, A., Jammer, A., Krbez, P., van der Graaff, E., Pfeifhofer, H., \& Roitsch, T. (2014). A rapid phytohormone and phytoalexin screening method for physiological phenotyping. Molecular Plant, 7, 10531056.

Grubbs, F. (1969). Procedures for detecting outlying observations in samples. Technometrics, 11, 1-21.

Guan, L., Tayengwa ,R., Cheng, Z. M., Peer, W. A., Murphy, A. S., \& Zhao, M. (2019). Auxin regulates adventitious root formation in tomato cuttings. BMC Plant Biology, 19, 435.

Gutierrez, L., Bussell, J.D., Pacurar, D.I., Schwambach, J., Pacurar, M., \& Bellini C. (2009). Phenotypic plasticity of adventitious rooting in Arabidopsis is controlled by complex regulation of AUXIN RESPONSE FACTOR transcripts and microRNA abundance. The Plant Cell, 21, 3119-3132. 
Heyman, J., Cools, T., Canher, B., Shavialenka, S., Traas, J., Vercauteren, I., Van den Daele, H., Persiau, G., De Jaeger, G., Sugimoto, K., \& De Veylder, L. (2016). The heterodimeric transcription factor complex ERF115-PAT1 grants regeneration competence. Nature Plants, 2, 16165.

Huang, A., Wang, Y., Liu, Y., Wang, G., She, X. (2020). Reactive oxygen species regulate auxin levels to mediate adventitious root induction in Arabidopsis hypocotyl cuttings. Jounal of Integrative Plant Biology, 62, 912-926.

Hilo, A., Shahinnia, F., Druege, U., Franken, P., Melzer, M., Rutten, T., Von Wirén, N., \& Hajirezaei, M. R. (2017). A specific role of iron in promoting meristematic cell division during adventitious root formation. Journal of Experimental Botany, 68, 4233-4247.

Hoermayer, L., Montesinos, J. C., Marhava, P., Benková, E., Yoshida, S., \& Friml, J. (2020). Wounding-induced changes in cellular pressure and localized auxin signalling spatially coordinate restorative divisions in roots. Proceedings of the National Academy of Sciences, 117, 15322-15331.

Hošek, P., Kubeš, M., Laňková, M., Dobrev, P. I., Klíma, P., Kohoutová, M., Petrášek, J., Hoyerová, K., Jiřina, M., \& Zažímalová, E. (2012). Auxin transport at cellular level: new insights supported by mathematical modelling. Journal of Experimental Botany, 63, 3815-3827.

Hu X, \& Xu L. (2016). Transcription factors WOX11/12 directly activate WOX5/7 to promote root primordia initiation and organogenesis. Plant Physiology, 172, 2363-2373.

Ivanchenko, M. G., Zhu, J., Wang ,B., Medvecká, E., Du, Y., Azzarello, E., Mancuso, S., Megraw, M., Filichkin, S., Dubrovsky, J.G., Friml, J., \& Geisler, M. (2015). The cyclophilin a DIAGEOTROPICA gene affects auxin transport in both root and shoot to control lateral root formation. Development, 142, 712721. 
Klopotek, Y., Franken, P., Klaering, H. P., Fischer, K., Hause, B., Hajirezaei, M. R., \& Druege, U. (2016). A higher sink competitiveness of the rooting zone and invertases are involved in dark stimulation of adventitious root formation in Petunia hybrida cuttings. Plant Science, 243, 10-22.

Kobayashi, M., Nagasaki, H., Garcia, V., Just, D., Bres, C., Mauxion, J-P., Le Paslier, M-C., Brunel, D., Suda, K., Minakuchi, Y., Toyoda, A., Fujiyama, A., Toyoshima, H., Suzuki, T., Igarashi, K., Rothan, C., Kaminuma, E., Nakamura, Y., Yano, K., \& Aoki, K. (2014). Genome-wide analysis of intraspecific DNA polymorphism in 'Micro-Tom', a model cultivar of tomato (Solanum lycopersicum). Plant and Cell Physiology, 55, 445-454.

Kurihara, D., Mizuta, Y., Sato, Y., Higashiyama, T. (2015). ClearSee: a rapid optical clearing reagent for whole-plant fluorescence imaging. Development, 142, 4168-4179.

Lakehal, A., \& Bellini, C. (2019). Control of adventitious root formation: insights into synergistic and antagonistic hormonal interactions. Physiologia Plantarum, 165, 90-100.

Lanková, M., Smith, R. S., Pesek, B., Kubes, M., Zazímalová, E., Petrásek, J., \& Hoyerová, K. (2010). Auxin influx inhibitors 1-NOA, 2-NOA, and CHPAA interfere with membrane dynamics in tobacco cells. Journal of Experimental Botany, 61, 3589-3598.

Lee, H. W., Cho, C., Pandey, S. K., Park, Y., Kim, M. J., \& Kim, J. (2019). LBD16 and LBD18 acting downstream of ARF7 and ARF19 are involved in adventitious root formation in Arabidopsis. BMC Plant Biology, 19, 46.

Liu, J., Sheng, L., Xu, Y., Li, J., Yang, Z., Huang, H., \& Xu, L. (2014). WOX11 and 12 are involved in the first-step cell fate transition during de novo root organogenesis in Arabidopsis. The Plant Cell, 26, 1081-1093.

Livak, K. J., \& Schmittgen, T. D. (2001). Analysis of relative gene expression data using real-time quantitative PCR and the $2^{-\Delta \Delta C}$ T method. Methods, 25, 402-408. 
Ludwig-Müller, J., Vertocnik, A., \& Town, C. D. (2005). Analysis of indole-3butyric acid-induced adventitious root formation on Arabidopsis stem segments. Journal of Experimental Botany, 56, 2095-2105.

Lu, Y., Xie, L., \& Chen, J. (2012). A novel procedure for absolute real-time quantification of gene expression patterns. Plant Methods, 8, 9.

Matosevich, R., Cohen, I., Gil-Yarom, N., Modrego, A., Friedlander-Shani, L., Verna, C., Scarpella, E., \& Efroni, I. (2020). Local auxin biosynthesis is required for root regeneration after wounding. Nature Plants, 6, 1020-1030.

Mignolli, F., Mariotti, L., Picciarelli, P., \& Vidoz, M. L. (2017). Differential auxin transport and accumulation in the stem base lead to profuse adventitious root primordia formation in the aerial roots (aer) mutant of tomato (Solanum lycopersicum L.). Journal of Plant Physiology, 213, 55-65.

Negi, S., Sukumar, P., Liu, X., Cohen, J. D., \& Muday, G. K. 2010. Genetic dissection of the role of ethylene in regulating auxin-dependent lateral and adventitious root formation in tomato. The Plant Journal, 61, 3-15.

Oh, K. C., Ivanchenko, M. G., White, T. J., \& Lomax, T. L. (2006). The DIAGEOTROPICA gene of tomato encodes a cyclophilin: a novel player in auxin signaling. Planta, 224, 133-144.

Pacurar, D.I., Perrone, I., \& Bellini, C. (2014). Auxin is a central player in the hormone cross-talks that control adventitious rooting. Physiologia Plantarum, 151, 83-96.

Pařízková, B., Pernisová, M., \& Novák, O. (2017). What has been seen cannot be unseen - detecting auxin in vivo. International Journal of Molecular Sciences, 18, 2736.

Rakusová, H., Abbas, M., Han, H., Song, S., Robert, H. S., \& Friml, J. (2016). Termination of shoot gravitropic responses by auxin feedback on PIN3 polarity. Current Biology, 26, 3026-3032. 
Rakusová, H., Gallego-Bartolomé, J., Vanstraelen, M., Robert, H. S., Alabadí, D., Blázquez, M. A., Benková, E., \& Friml, J. (2011). Polarization of PIN3dependent auxin transport for hypocotyl gravitropic response in Arabidopsis thaliana. The Plant Journal, 67, 817-826.

Rothan, C., Bres, C., Garcia, V., \& Just, D. (2016). Tomato resources for functional genomics. In: Causse, M., Giovannoni, J., Bouzayen, M., Zouine M. (eds) The Tomato Genome. Compendium of Plant Genomes. Springer, Berlin, Heidelberg.

Rothan, C., Diouf, I., \& Causse, M. (2019). Trait discovery and editing in tomato. The Plant Journal, 97, 73-90.

Shikata, M., Hoshikawa, K., Ariizumi, T., Fukuda, N., Yamazaki, Y., \& Ezura, H. (2016). TOMATOMA update: phenotypic and metabolite information in the Micro-Tom Mutant resource. Plant and Cell Physiology, 57, e11.

Smetana, O., Mäkilä, R., Lyu, M., Amiryousefi, A., Sánchez-Rodríguez, F., Wu, M. F., Solé-Gil, A., Leal Gavarrón, M., Siligato, R., Miyashima, S., Roszak, P., Blomster, T., Reed, J. W., Broholm, S., \& Mähönen, A. P. (2019). High levels of auxin signalling define the stem-cell organizer of the vascular cambium. Nature, 565, 485-489.

Sorin, C., Bussell, J. D., Camus, I., Ljung, K., Kowalczyk, M., Geiss, G., McKhann, H., Garcion, C., Vaucheret, H., Sandberg, G., \& Bellini, C. (2005). Auxin and light control of adventitious rooting in Arabidopsis require ARGONAUTE1. The Plant Cell, 17, 1343-1359.

Su, S-H., Gibbs, N. M., Jancewicz, A.L., \& Masson, P. H. (2017). Molecular mechanisms of root gravitropism. Current Biology, 27, R964-R972.

Sukumar, P., Maloney, G. S., \& Muday, G. K. (2013). Localized induction of the ATP-binding cassette B19 auxin transporter enhances adventitious root formation in Arabidopsis. Plant Physiology, 162, 1392-1405.

Teale, W., \& Palme, K. (2018). Naphthylphthalamic acid and the mechanism of polar auxin transport. Journal of Experimental Botany, 69, 303-312. 
Tsugafune, S., Mashiguchi, K., Fukui, K., Takebayashi, Y., Nishimura, T., Sakai, T., Shimada, Y., Kasahara, H., Koshiba, T., \& Hayashi, K. I. (2017). Yucasin $\mathrm{DF}$, a potent and persistent inhibitor of auxin biosynthesis in plants. Scientific Reports, 7, 13992.

Tyburski, J., \& Tretyn, A. (2004). The role of light and polar auxin transport in root regeneration from hypocotyls of tomato seedling cuttings. Plant Growth Regulation, 42, 39-48.

Vidoz, M. L., Loreti, E., Mensuali, A., Alpi, A., \& Perata, P. (2010). Hormonal interplay during adventitious root formation in flooded tomato plants. The Plant Journal, 63, 551-562.

Villacorta-Martín, C., Sánchez-García, A. B., Villanova, J., Cano, A., van de Rhee, M., de Haan, J., Acosta, M., Passarinho, P., \& Pérez-Pérez, J. M. (2015). Gene expression profiling during adventitious root formation in carnation stem cuttings. BMC Genomics, 16, 789.

Welander, M., Geier, T., Smolka, A., Ahlman, A., Fan, J., \& Zhu, L.H. (2014). Origin, timing, and gene expression profile of adventitious rooting in Arabidopsis hypocotyls and stems. American Journal of Botany, 101, 255-266. Wu, J., Peng, Z., Liu, S., He, Y., Cheng, L., Kong, F., Wang, J., \& Lu, G. (2012). Genome-wide analysis of Aux/IAA gene family in Solanaceae species using tomato as a model. Molecular Genetics and Genomics, 287, 295-311.

Yang, H., Klopotek, Y., Hajirezaei, M. R., Zerche, S., Franken, P., \& Druege, U. (2019). Role of auxin homeostasis and response in nitrogen limitation and dark stimulation of adventitious root formation in petunia cuttings. Annals of Botany, 124, 1053-1066.

Ye, B-B., Shang, G. D., Pan, Y., Xu, Z. G., Zhou, C. M., Mao, Y. B., Bao, N., Sun, L., Xu, T., \& Wang, J. W. (2020). AP2/ERF transcription factors integrate age and wound signals for root regeneration. The Plant Cell, 32, 226-241.

Zerche, S., Haensch, K-T. T., Druege, U., Hajirezaei, M. R. 2016. Nitrogen remobilisation facilitates adventitious root formation on reversible dark-induced carbohydrate depletion in Petunia hybrida. BMC Plant Biology, 16, 219. 
Zhang, G., Zhao, F., Chen, L., Pan, Y., Sun, L., Bao, N., Zhang, T., Cui, C-X., Qiu, Z., Zhang, Y, Yang, L., \& Xu, L. (2019). Jasmonate-mediated wound signalling promotes plant regeneration. Nature Plants, 5, 491-497.

Zhang, J., Chen, R., Xiao, J., Qian, C., Wang, T., Li, H., Ouyang, B., \& Ye, Z. (2007). A single-base deletion mutation in SIIAA9 gene causes tomato (Solanum lycopersicum) entire mutant. Journal of Plant Research, 120, 671-678.

Zhou, W., Lozano-Torres, J. L., Blilou, I., Zhang, X., Zhai, Q., Smant, G., Li, C., $\&$ Scheres, B. (2019). A jasmonate signaling network activates root stem cells and promotes regeneration. Cell, 177, 942-946.

Zouine, M., Fu, Y., Chateigner-Boutin, A. L., Mila, I., Frasse, P., Wang, H., Audran, C., Roustan, J. P., \& Bouzayen, M. (2014). Characterization of the tomato ARF gene family uncovers a multi-levels post-transcriptional regulation including alternative splicing. PLoS ONE, 9, e84203. 


\section{FIGURES}
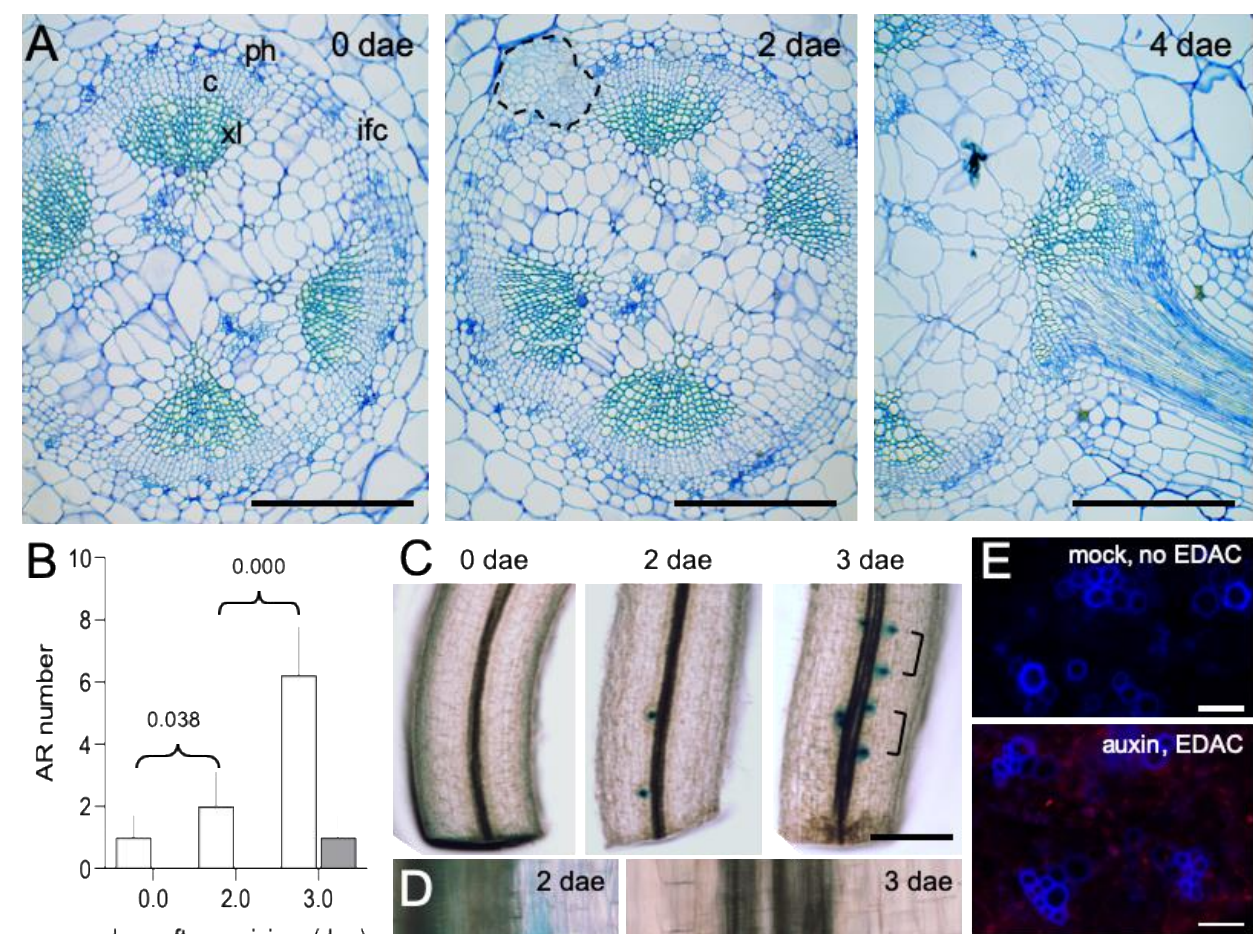

C 0 dae
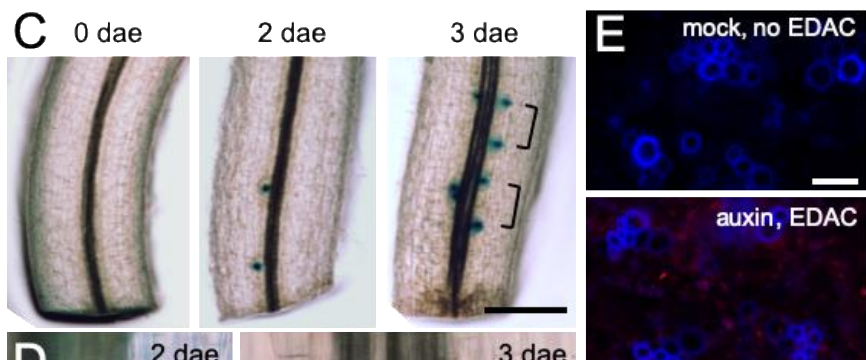
days after excision (dae)

$\square$ DR5 positive $\square$ emerged ARs
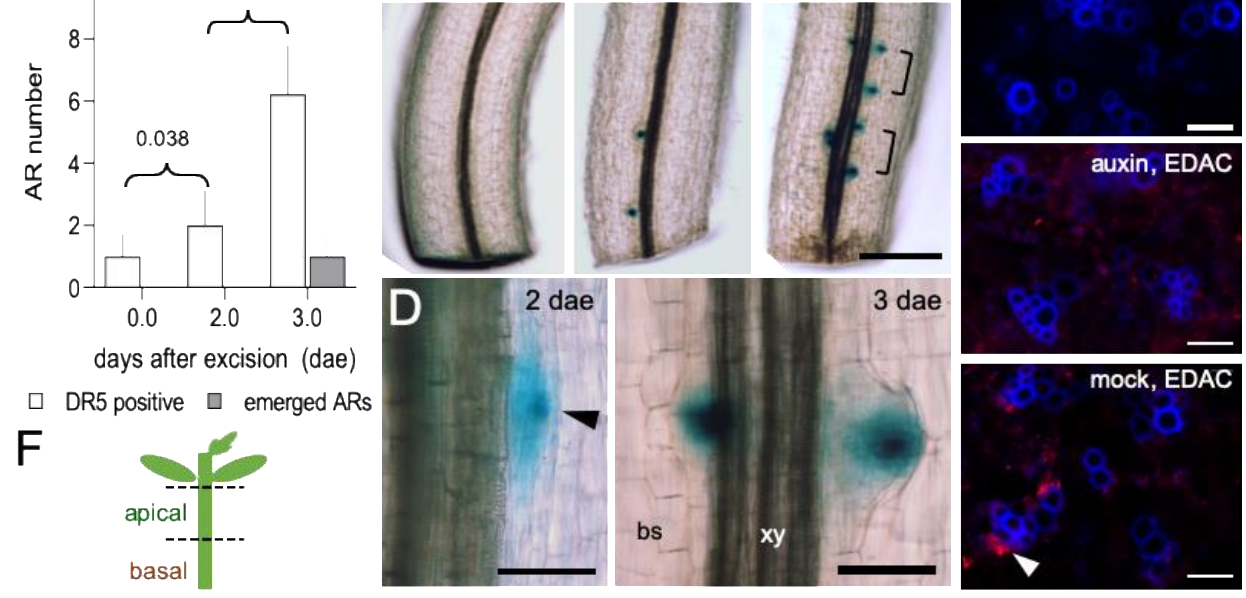

mock, EDAC
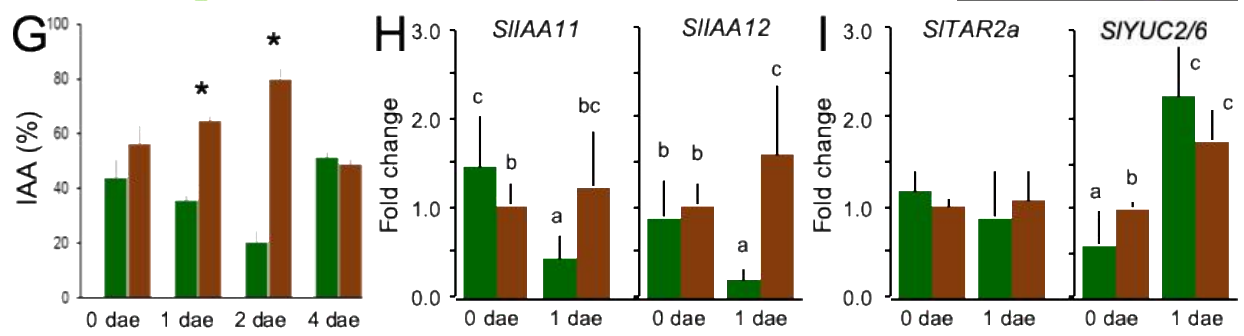

Fig. 1. A functional auxin gradient in the basal region of the hypocotyl correlates with wound-induced AR formation in tomato shoot explants. (A) Representative cross sections of the basal region of the hypocotyl during 
AR formation. Dotted lines surround an early AR primordia arising from the cambium next to the vascular bundle; c, cambium; ifc, interfascicular cambium; ph, phloem; xl, xylem. (B) Wound-induced AR number expressing DR5::GUS after whole root excision. (C, D) Representative images of GUS expression in the AR primordia of DR5::GUS lines. Black lines indicate regular spacing between adjacent AR primordia. (E) Immunolocalization of IAA near the xylem pole (white arrow) of the basal region of the shoot explants at 2 dae. (F) Diagram showing the different regions studied in G-I. (G) Percentage of IAA levels in the different hypocotyl regions during AR formation. (H, I) Relative expression of Aux/IAA-related $(\mathrm{H})$ and auxin biosynthesis (I) genes. Bars indicate normalized expression levels \pm SD with regards to the expression at the basal region at 0 dae. Letters/Asterisks indicate significant differences ( $\mathrm{p}$-value $<0.01$ ) between regions. Scale bars: $200 \mu \mathrm{M}$ (A, D, E) and $1 \mathrm{~mm}(\mathrm{C})$. 

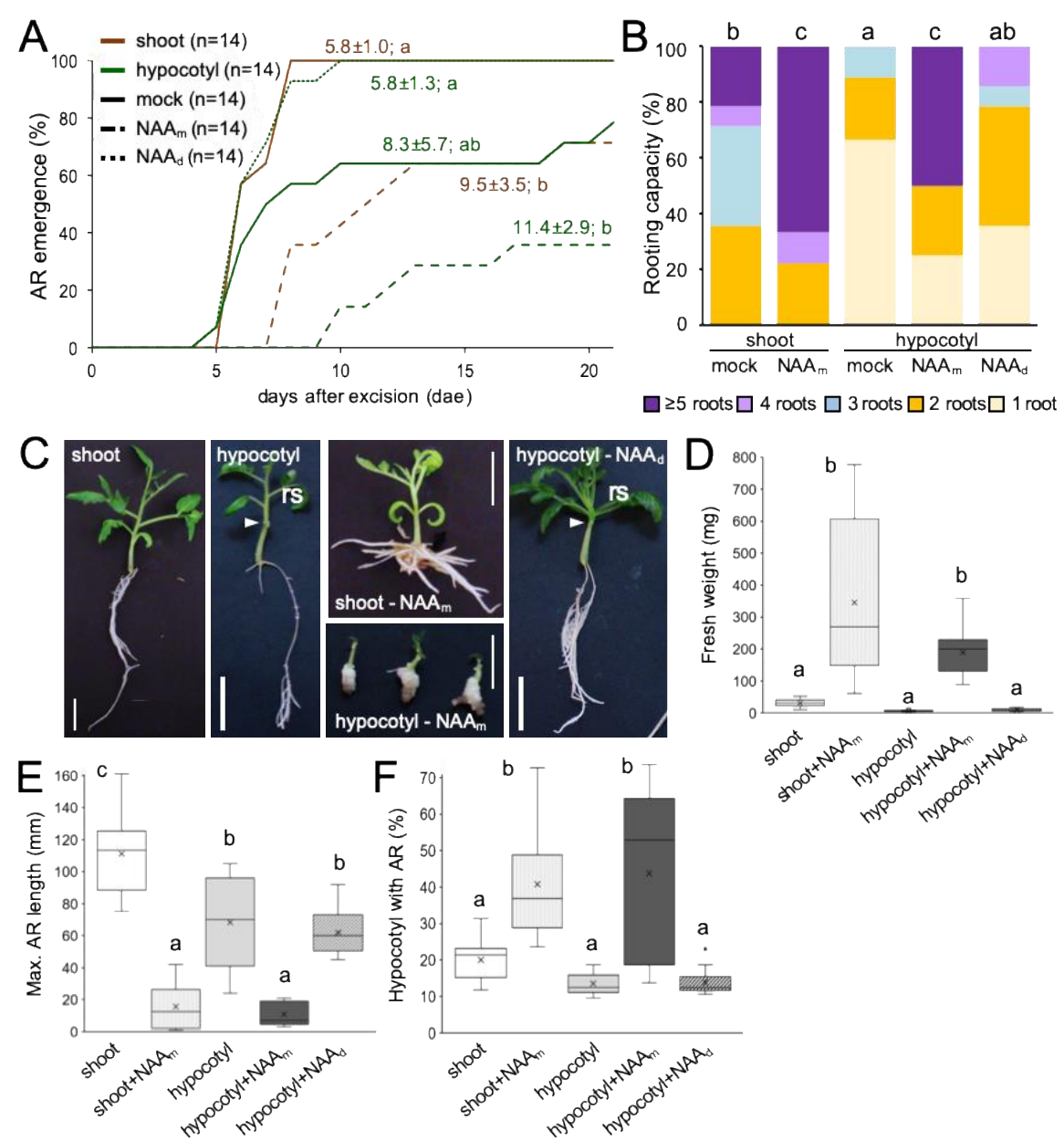

Fig. 2. Auxin effect on wound-induced AR formation in tomato shoot explants. (A) AR emergence of the studied explants. (B) Rooting capacity of 'Micro-Tom' shoot explants at 14 dae. (C) Representative images of rooted shoot explants. Arrow indicate the apical end of the hypocotyl explants; rs: regenerated shoot, NAAm: NAA added to the SGM; NAAd: NAA added to the distal end of the hypocotyl explants. (D) Fresh weight of the distal region of the shoot explants with ARs at 21 dae. (E) Maximum length of ARs at 21 dae. (F) Percentage of hypocotyl length with ARs at 21 dae. Letters indicate significant differences ( $p$-value $<0.01$ ) between the assayed conditions. Scale bars: $20 \mathrm{~mm}$. 

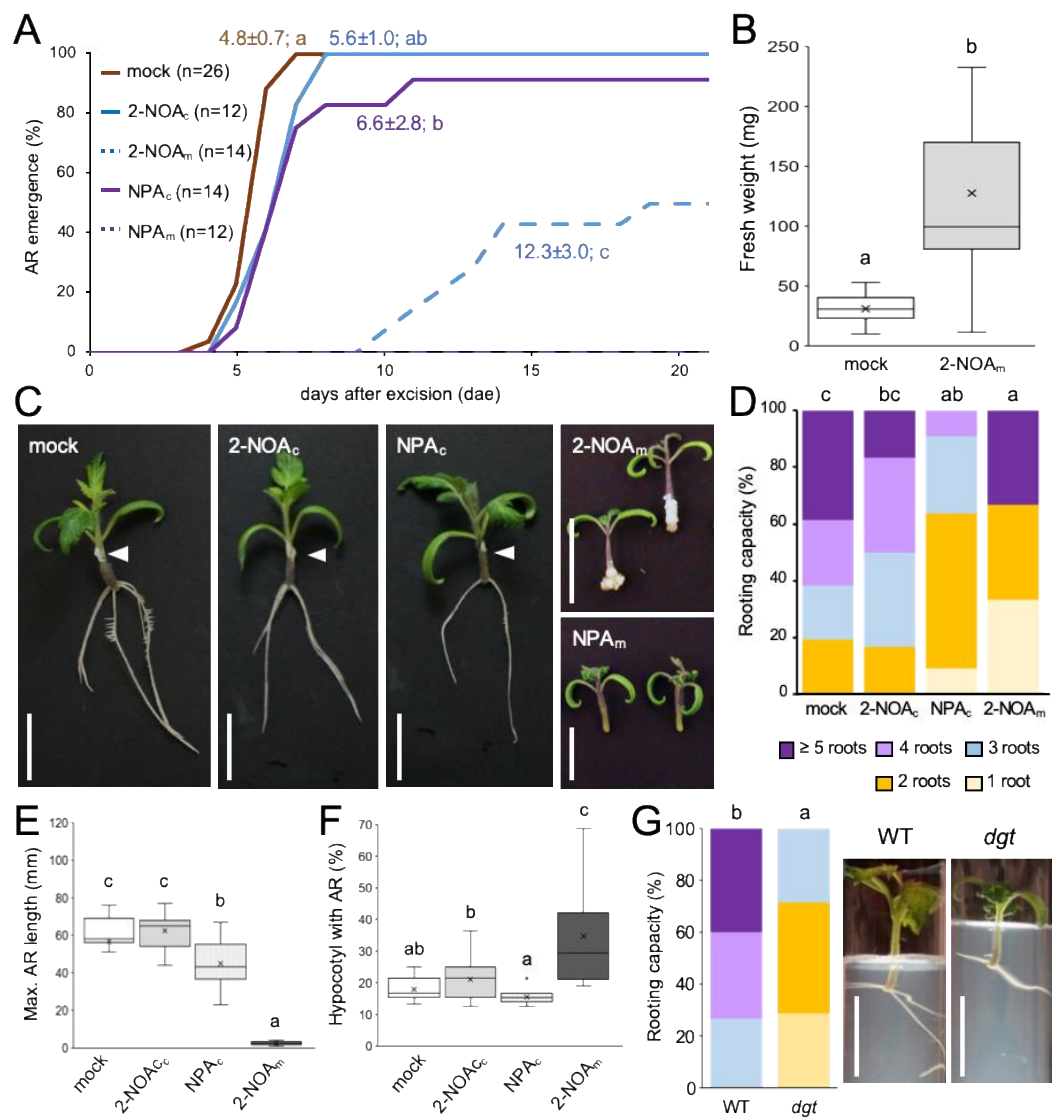

Fig. 3. Polar auxin transport is required for wound-induced AR formation in tomato shoot explants. (A) AR emergence of the studied explants. (B) Fresh weight of the AR formative region of the shoot explants at 21 dae. (C) Representative images of rooted shoot explants at 14 dae. The chemical treatments were applied on a lanolin collar (c; white arrow) or to the medium (m). (D, G left) Rooting capacity of shoot explants at 14 dae. (E) Maximum lengths of ARs at 14 dae. (F) Percentage of hypocotyl length with ARs at 14 dae. (G right) Representative images of rooted shoot explants at 14 dae in diageotropica (dgt) and 'Micro-Tom' (WT). Letters indicate significant differences ( $\mathrm{p}$-value<0.01) between treatments and genotypes. Scale bars: $20 \mathrm{~mm}$. 

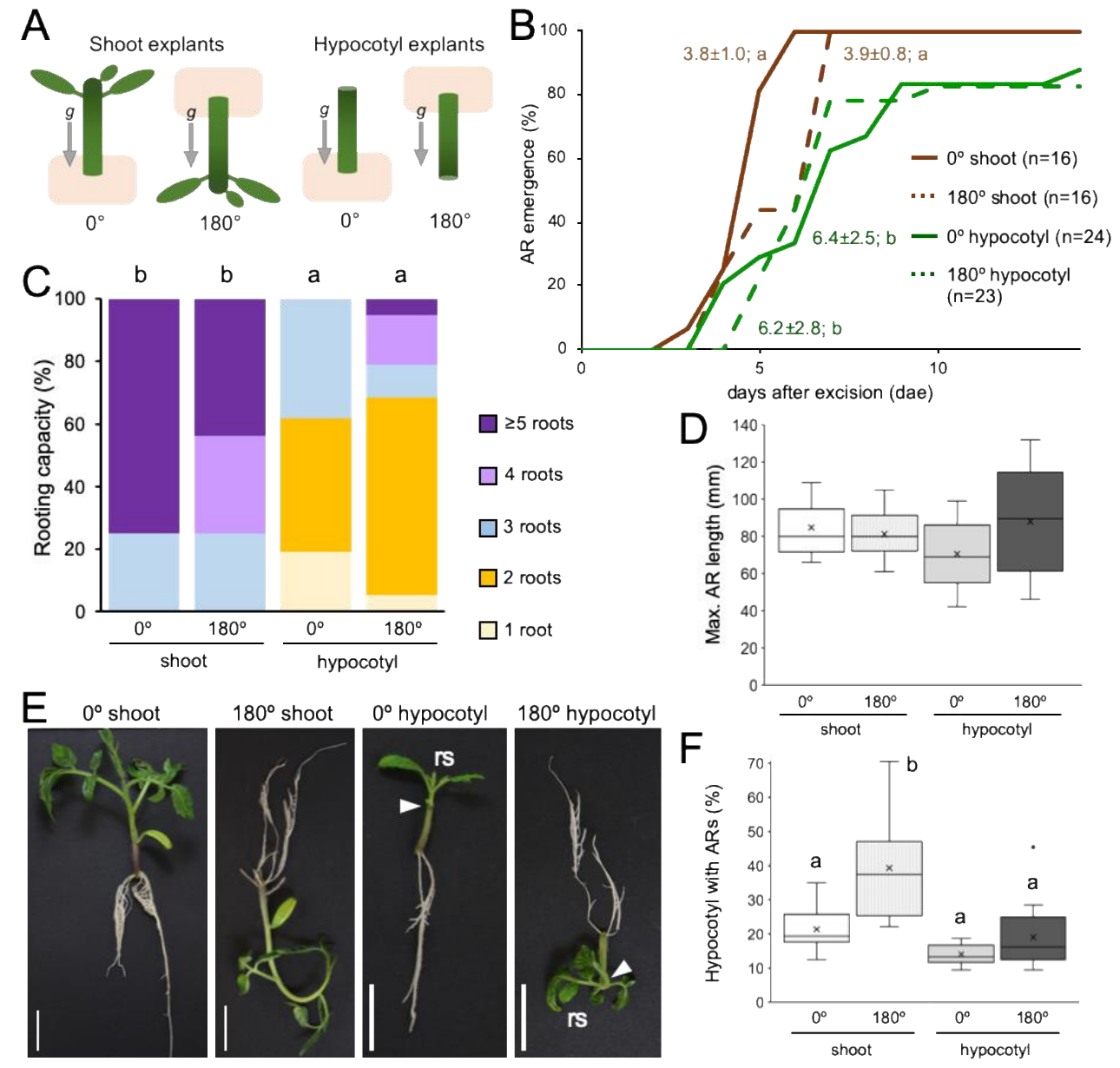

Fig. 4. Wound-induced AR formation in tomato explants is independent of the gravity vector. (A) Shoot or hypocotyl explants were oriented with $\left(0^{\circ}\right)$ or opposite $\left(180^{\circ}\right)$ the gravity vector. (B) AR emergence of the explants in the studied conditions. (C) Rooting capacity of 'Micro-Tom' explants at 14 dae. (D) Maximum lengths of ARs at 21 dae. (E) Representative images of rooted shoot explants at 21 dae. Arrows indicate the apical end of the hypocotyl explants; rs: regenerated shoot. (F) Percentage of hypocotyl lengths with functional ARs at 21 dae. Letters indicate significant differences (p-value<0.01) between treatments. Scale bars: $20 \mathrm{~mm}$. 

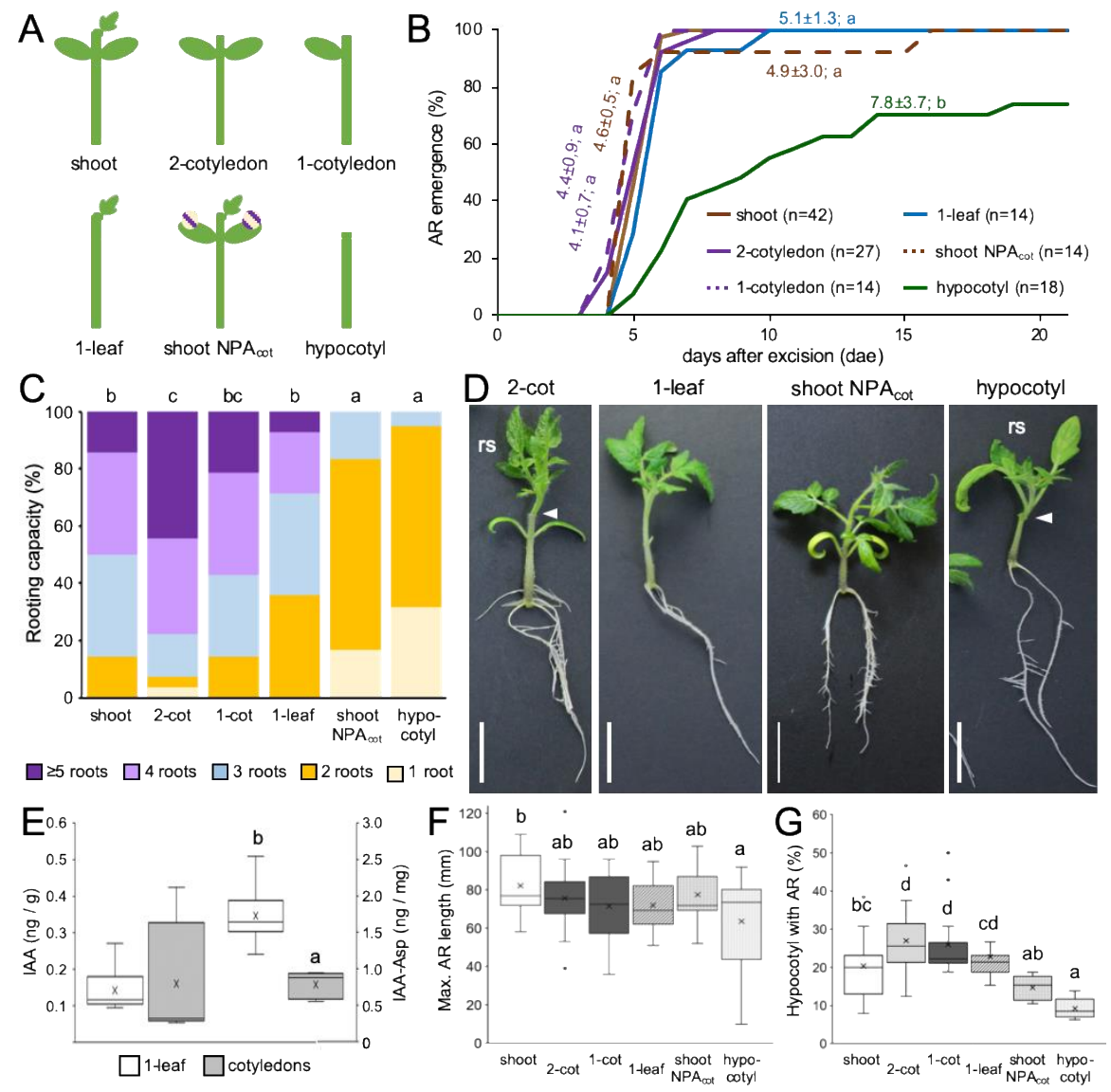

Fig. 5. Source of active auxin required for wound-induced $A R$ formation in tomato hypocotyl explants. (A) Diagram showing the different conditions assayed. (B) AR emergence of the studied explants. (C) Rooting capacity of 'Micro-Tom' shoot explants at 14 dae. (D) Representative images of rooted shoot explants at 21 dae. Arrows indicate the apical end of the hypocotyl explants; rs: regenerated shoot. (E) IAA and IAA-Asp levels in the shoot apex or the cotyledons in shoot explants at 21 dae. (F) Maximum lengths of ARs at 21 dae. (G) Percentage of hypocotyl length with ARs at 21 dae. Letters indicate significant differences $(p-v a l u e<0.01)$ between treatments. Scale bars: $20 \mathrm{~mm}$. 

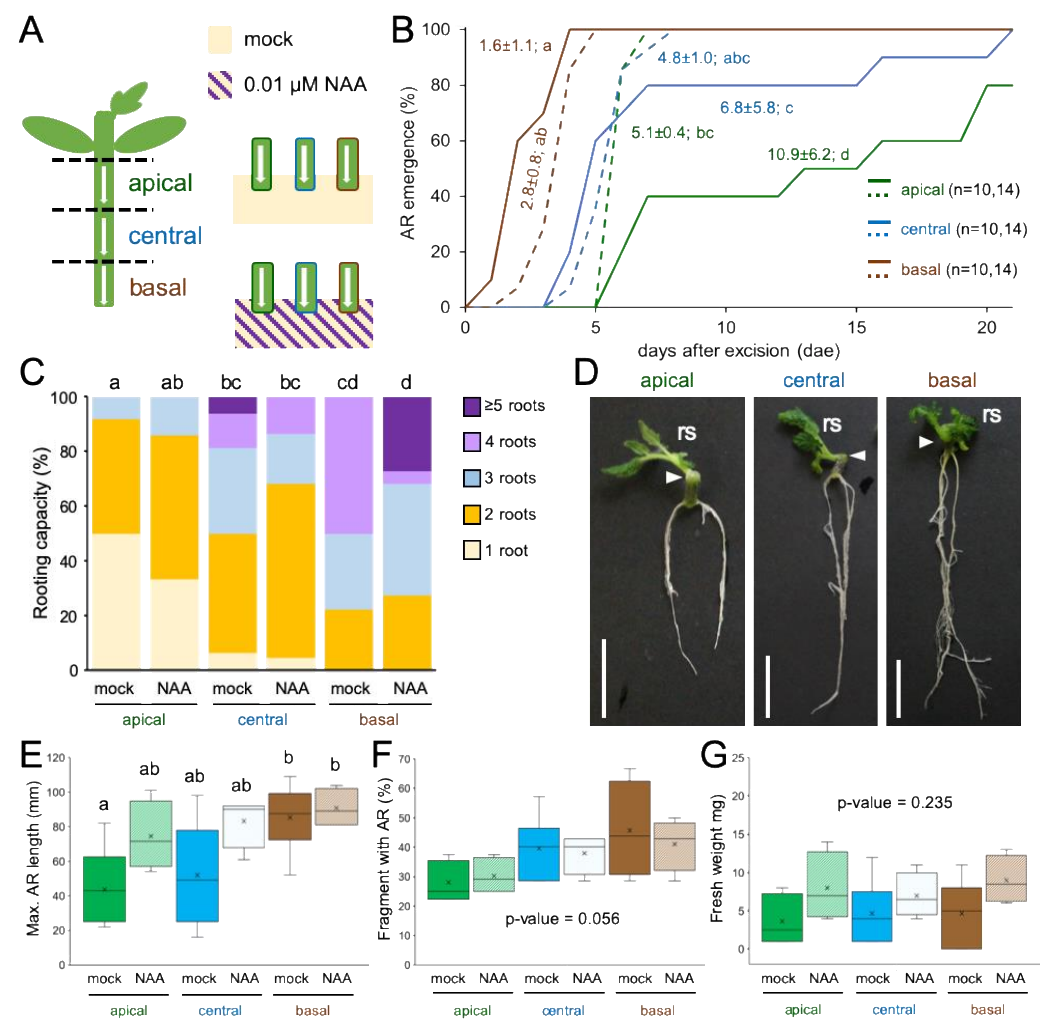

Fig. 6. Apical-basal gradient of auxin signaling within the hypocotyl influences wound-induced AR formation. (A) Diagram showing the different conditions assayed. White arrow indicates the internal tissue polarity. (B) AR emergence of the studied explants. Dotted lines indicate hypocotyl explants incubated with $0.01 \mu \mathrm{M}$ NAA. (C) Rooting capacity of 'Micro-Tom' shoot explants at 14 dae in the different conditions assayed. (D) Representative images of rooted shoot explants at 21 dae in the studied regions without exogenous auxin treatment. Arrows indicated the apical end of the hypocotyl explants; rs: regenerated shoot. (E) Maximum lengths of ARs at 21 dae. (F) Percentage of hypocotyl length with ARs at 21 dae. (G) Fresh weight of the distal end of the explants with AR formative region. Letters indicate significant differences $(p$-value<0.01) between the experimental conditions tested. Scale bars: $20 \mathrm{~mm}$. 

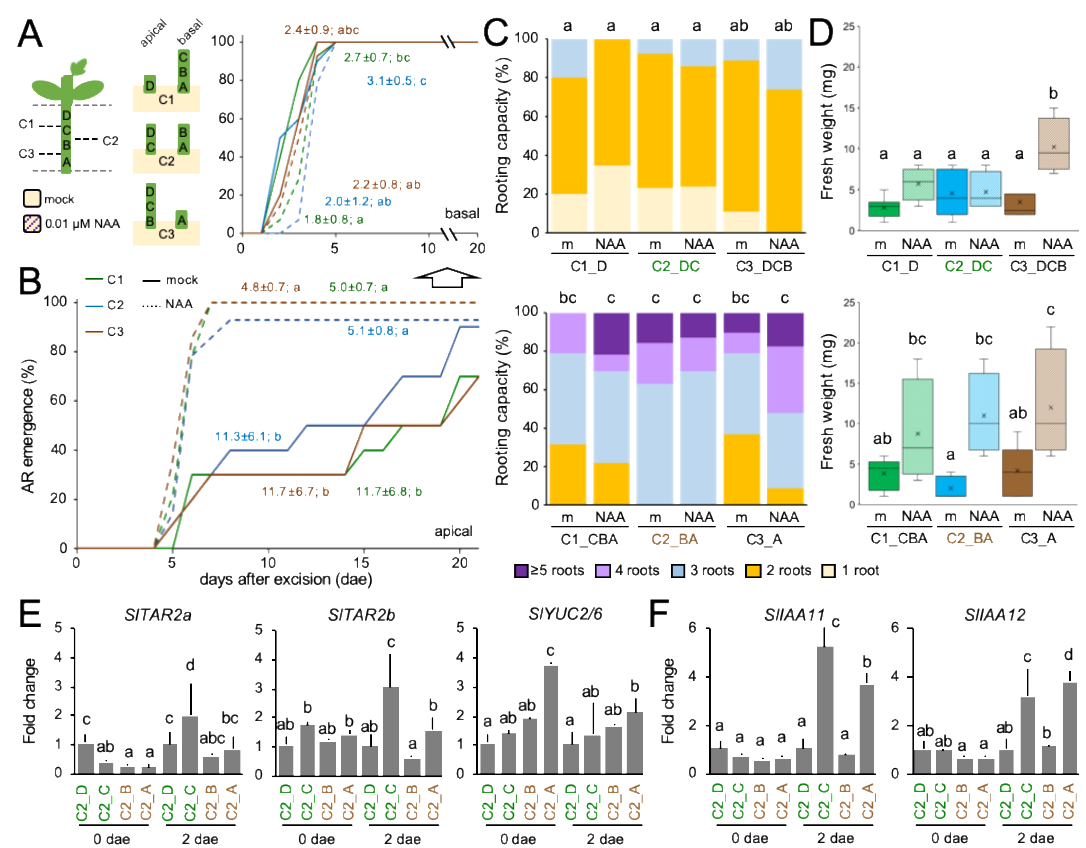

SIYUC2/6
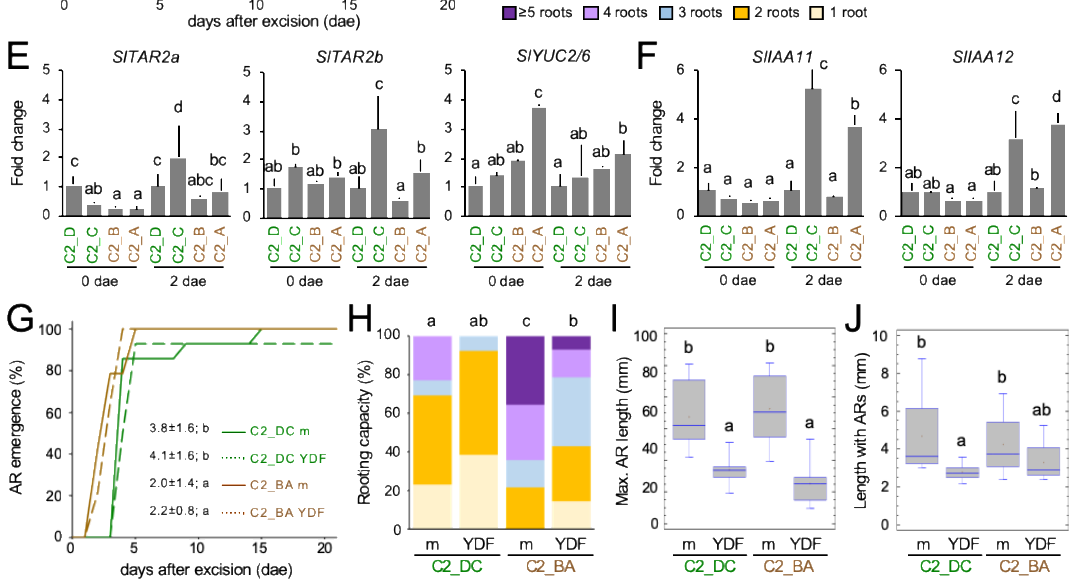

Fig 7. Apical-basal polarity within the hypocotyl regulates woundinduced AR formation. (A) Diagram showing the different conditions assayed. (B, G) AR emergence of the explants in the studied conditions. YDF: $50 \mu \mathrm{M}$ yucasin DF; m: mock. (C, H) Rooting capacity of 'Micro-Tom' shoot explants at 14 dae. (C) Upper graph: apical; lower graph: basal. (D) Fresh weight of the distal end of the explants with ARs at 21 dae. (E, F) Relative expression of auxin biosynthesis (E) and auxin responsive (F) genes. Bars indicate normalized expression levels \pm SD with regards to the apical region of the hypocotyl (C2_A) at a given time. (I) Maximum lengths of ARs at 21 dae. (J) Hypocotyl length with ARs at 21 dae. Letters indicate significant differences ( $p$-value<0.01) between the studied conditions. 

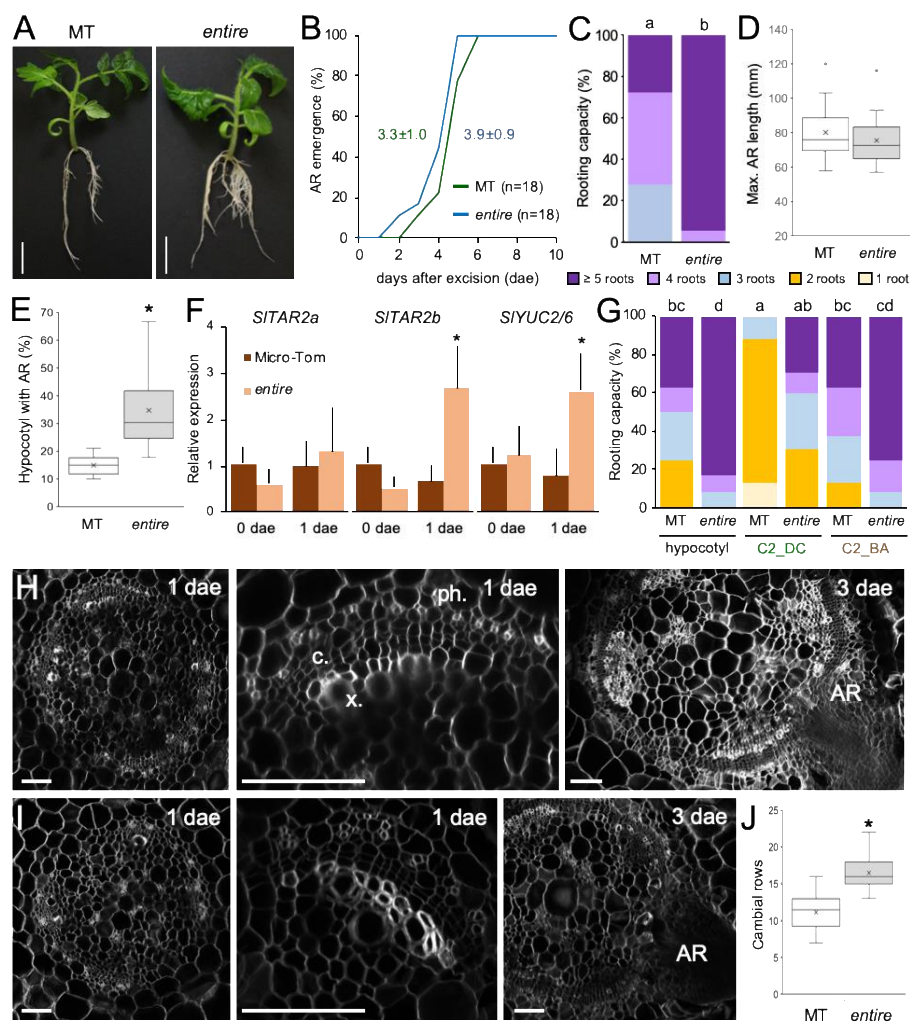

Fig. 8. Auxin response in the basal region of the hypocotyl is required for wound-induced $A R$ formation in tomato shoot explants. (A) Representative images of rooted shoot explants at 21 dae. (B) AR emergence of the studied explants. (C, G) Rooting capacity of wild-type (WT) and entire shoot and hypocotyl explants at 14 dae. (D) Maximum lengths of ARs at 21 dae. (E) Percentage of hypocotyl lengths with ARs at 21 dae. (F) Relative expression of auxin biosynthesis genes. Bars indicate normalized expression levels $\pm \mathrm{SD}$ with regards to the WT expression in the basal region at 0 dae. $(\mathrm{H}, \mathrm{I})$ Representative cross sections of shoot explants of entire $(\mathrm{H})$ and WT (I) at 1 and 3 dae. (J) Number of cambial rows per vascular bundle in hypocotyl cross sections of WT and entire at 1 dae. Letters $(C, G)$ or asterisks $(\mathrm{E}, \mathrm{F}, \mathrm{J})$ indicate significant differences ( $\mathrm{p}$-value $<0.01)$ between genotypes and/or conditions tested. Scale bars: $20 \mathrm{~mm}(\mathrm{~A})$ and $100 \mu \mathrm{M}(\mathrm{H}, \mathrm{I})$. 

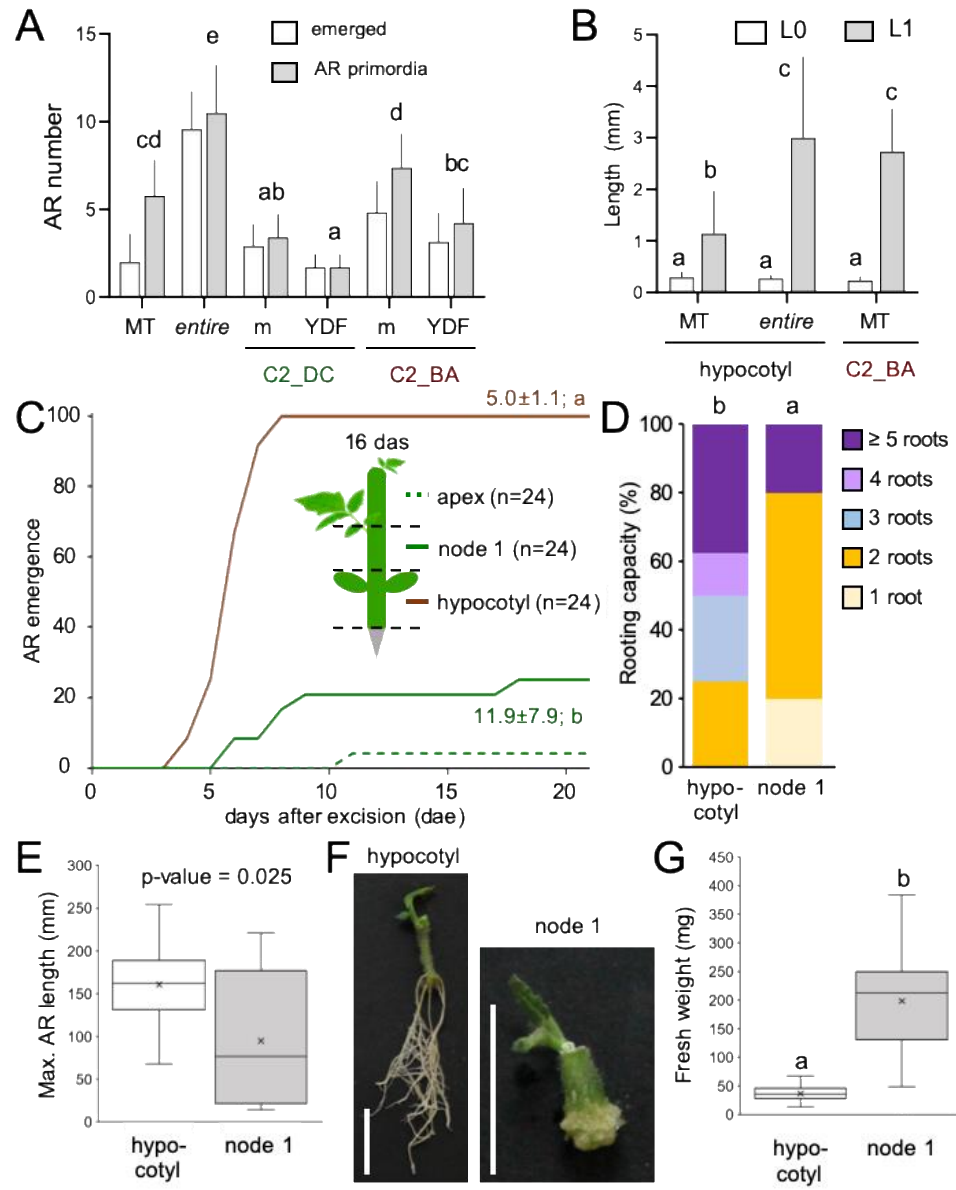

H TO (9 das)

$F$ hypocotyl

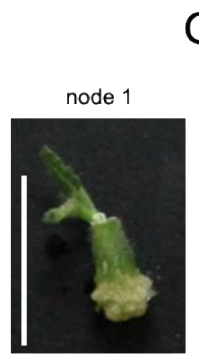

G ${ }^{45}$
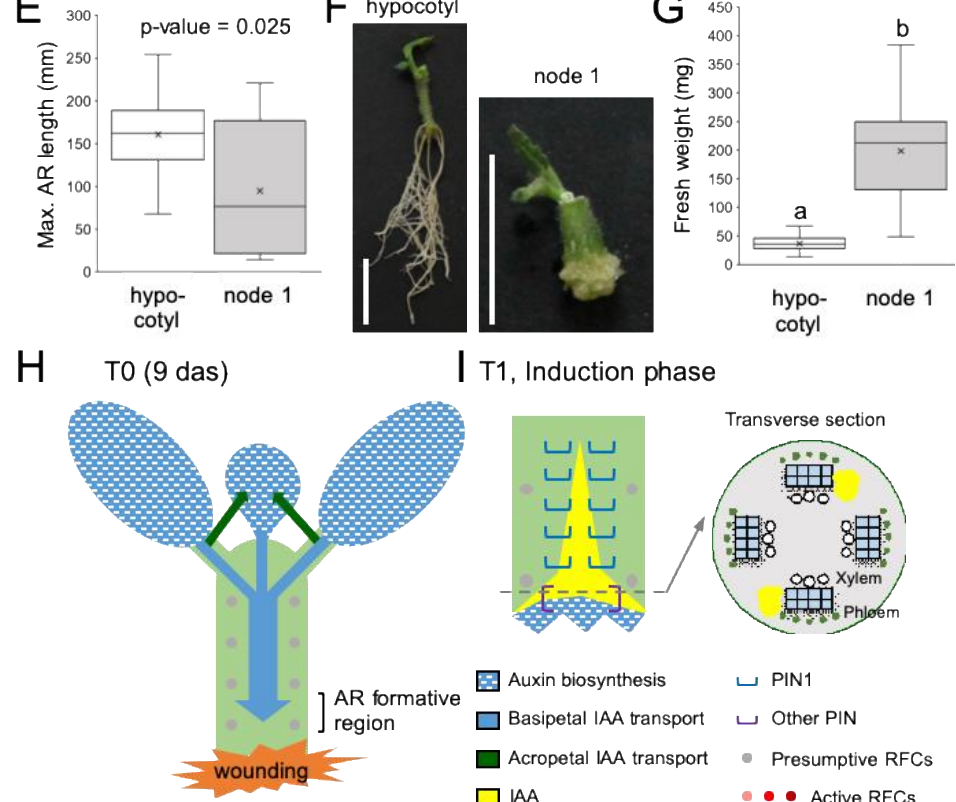

I T1, Induction phase

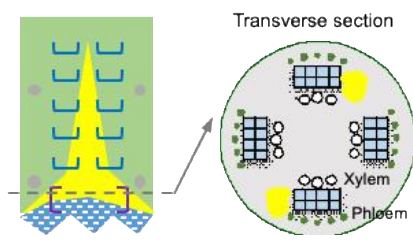

Auxin biosynthesis

PIN1

$\square$ Basipetal IAA transport

$\sqcup$ Other PIN

$\square$ Acropetal IAA transport

- Presumptive RFCs

$\square$ IAA

- - Active RFCs
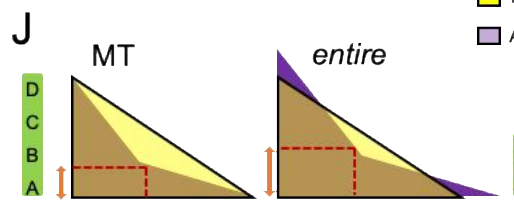

Auxin respons

$\boxplus$ Cambium

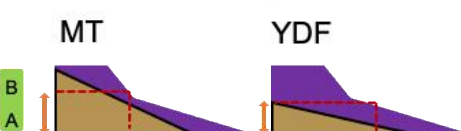


Fig. 9. A developmental gradient of auxin response is required for wound-induced $A R$ formation in tomato. (A) Number of AR primordia in different conditions; m: mock, YDF: $50 \mu \mathrm{M}$ yucasin DF. (B) Length of the basal region of the hypocotyl with no AR formation (L0) or with AR formation (L1). (C) AR emergence of the studied explants, as indicated in the diagram. (D) Rooting capacity of hypoctyl and node 1 explants at 14 dae. (E) Maximum lengths of ARs. (F) Representative images of explants at 28 dae. Scale bars: $20 \mathrm{~mm}$. (G) Fresh weight of the distal end of the explants at 14 dae. Letters indicate significant differences ( $p$-value $<0.01)$ between the assayed conditions. (H-J) Model of wound-induced AR formation in tomato shoot explants. (H) IAA is produced in some shoot tissues (cotyledons and young leaves) and it is transported through the hypocotyl and into the primary root. Auxin produced in the cotyledons also contributes to the growth of young leaves. Immediately after whole root excision, wound-induced signaling is produced near the wound. RFCs: root founder cells. (I) In the AR formative region, the IAA levels in the vascular bundles increase due to basipetal auxin transport from the shoot and local auxin biosynthesis near the wound. AUX/LAX1 and PIN proteins actively contribute to the buildup of endogenous auxin gradients within the vascular bundles, where they activate RFCs within the cambium. (J) In hypocotyl explants, an internal pre-pattern of auxin sensitivity is already established, likely through regulation of Aux/IAA protein levels. The size of the AR formative region depends on a given threshold of IAA level, which is directly interpreted by the prepatterned auxin-response gradient. 


\section{SUPPORTING INFORMATION}
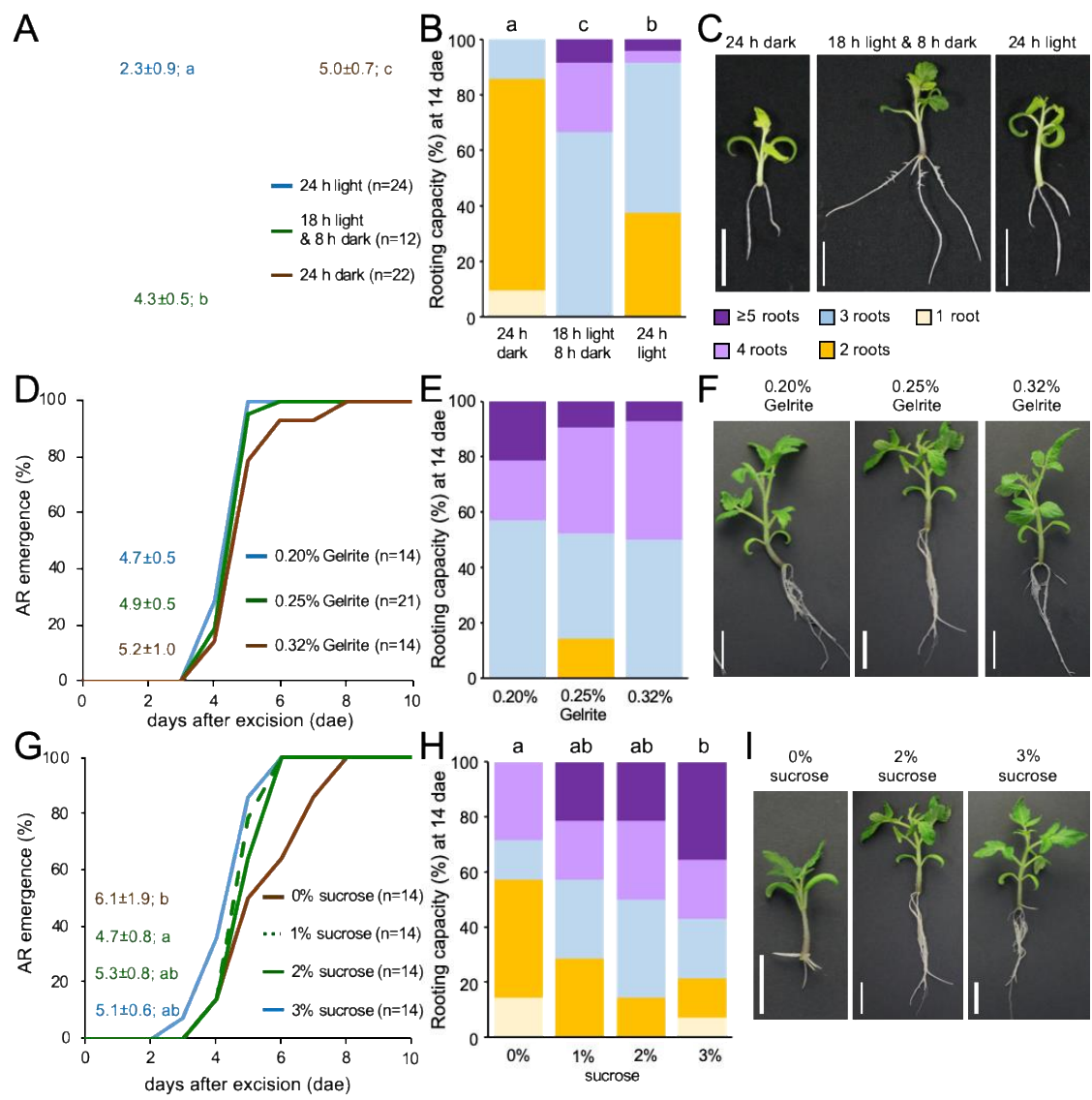

Fig. S1. Environmental dependence of wound-induced $A R$ formation in tomato shoot explants. Effect of light regime (A-C), gelling agent (D-F) or sucrose (G-I) on wound-induced AR formation in tomato shoot explants. (A, D, G) AR emergence of the explants in the studied conditions; numbers indicate average $\pm \mathrm{SD}$ of days for AR emergence. (B, E, H) Rooting capacity of 'Micro-Tom' shoot explants in the studied conditions. (C, F, I) Representative images of rooted shoot explants in the different conditions assayed. Letters indicate significant differences ( $\mathrm{p}$-value $<0.01$ ) between treatments. Scale bars: $20 \mathrm{~mm}$. 


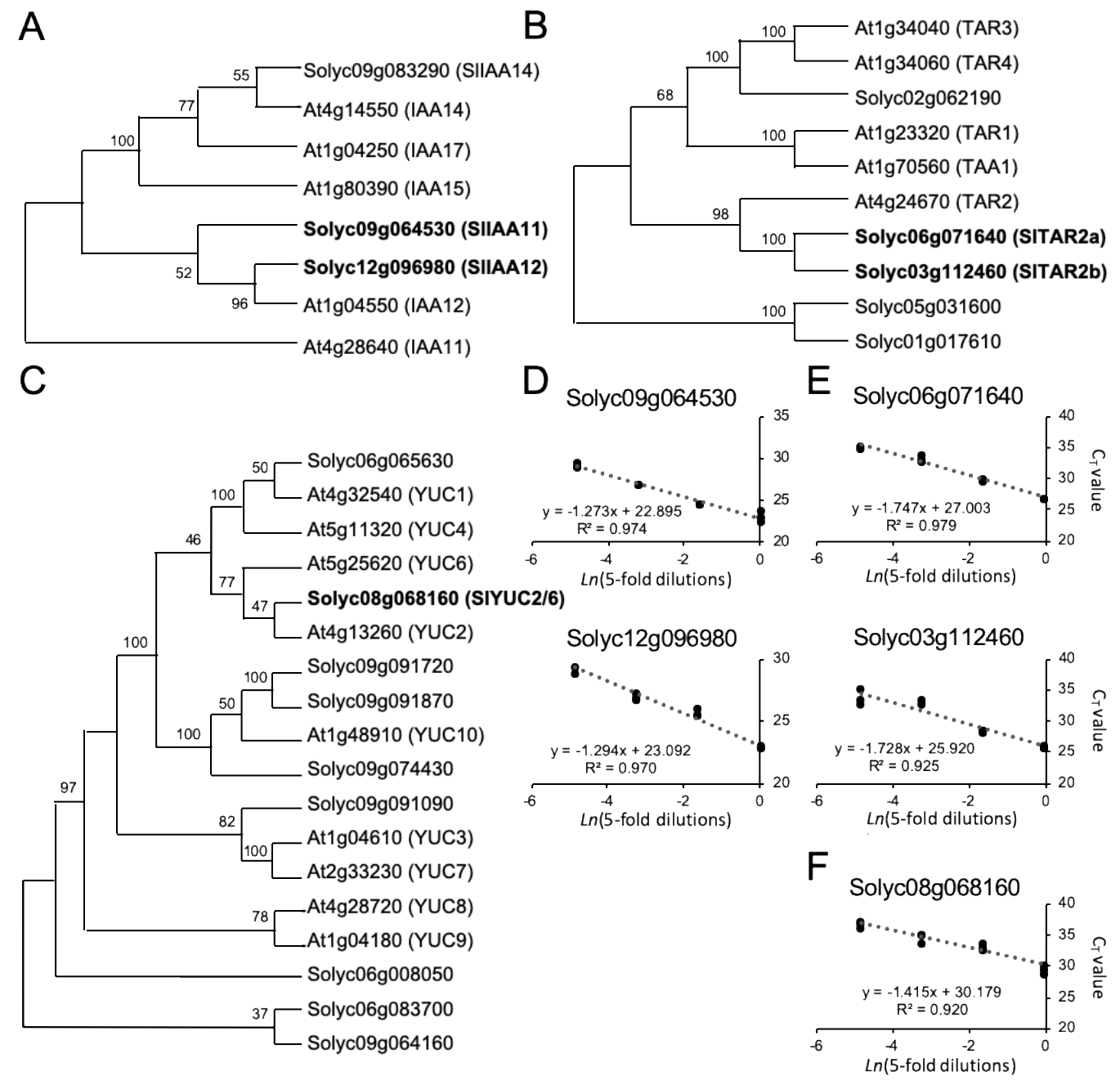

Fig. S2. Phylogenetic analysis of auxin biosynthesis genes in tomato and primer validation for real-time quantitative PCR. (A, B, C) Phylogenetic trees of a subset of Aux/IAA (A), TAA/TAR (B) and YUCCA (C) proteins. The trees were inferred from protein alignments by using the Maximum Likelihood method based on the Le Gascuel (2008) model. (D, E, F) Primer validation was performed for selected Aux/IAA (D), TAA/TAR (E) and YUC (F) genes. Each dot represents the relative expression data for a given sample. 

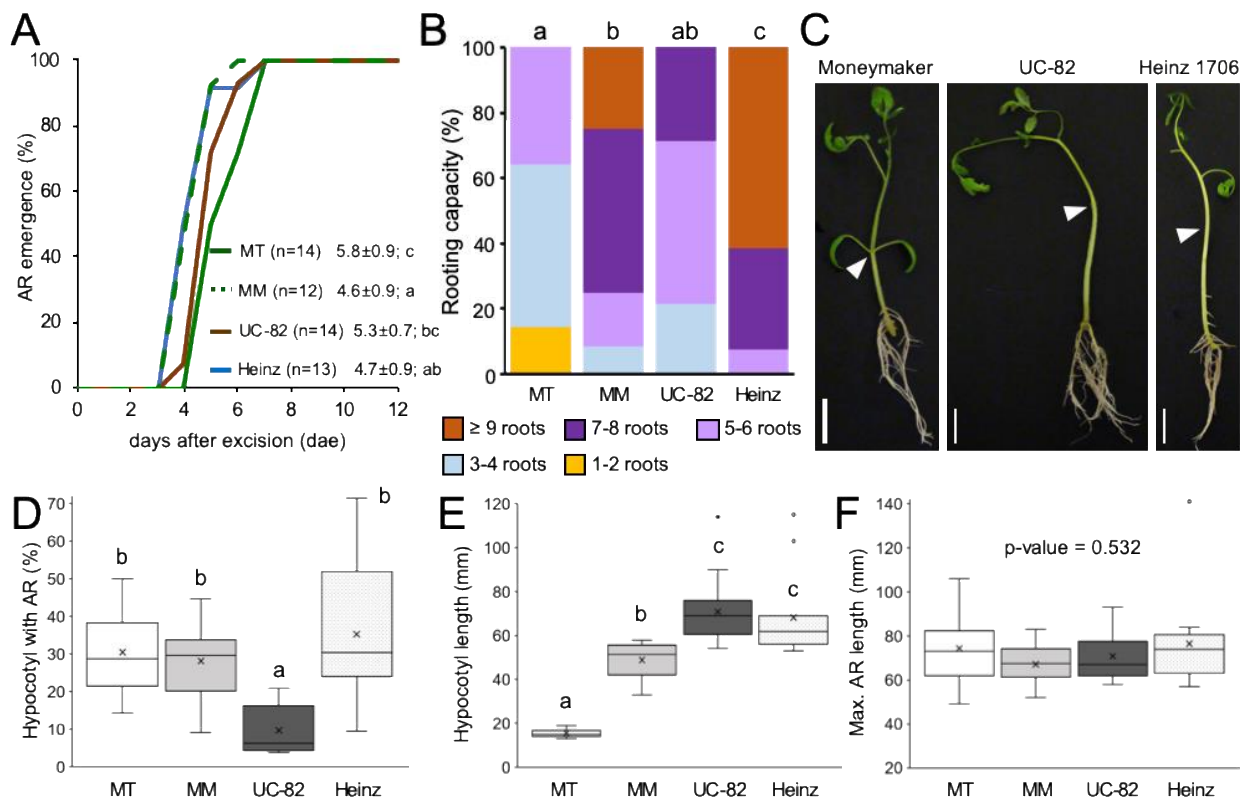

Fig. S3. Wound-induced AR formation in tomato shoot explants is genotype-dependent. (A) AR emergence of the studied explants. (B) Rooting capacity of shoot explants in the studied cultivars at 14 dae. (C) Representative images of rooted shoot explants at 14 dae in the studied cultivars. Arrowheads indicate the position of the cotyledons. (D) Percentage of hypocotyl length with ARs and (E) hypocotyl lengths at 14 dae. Letters indicate significant differences $(\mathrm{p}$-value $<0.01)$ between cultivars and conditions assayed. Scale bars: $20 \mathrm{~mm}$. 


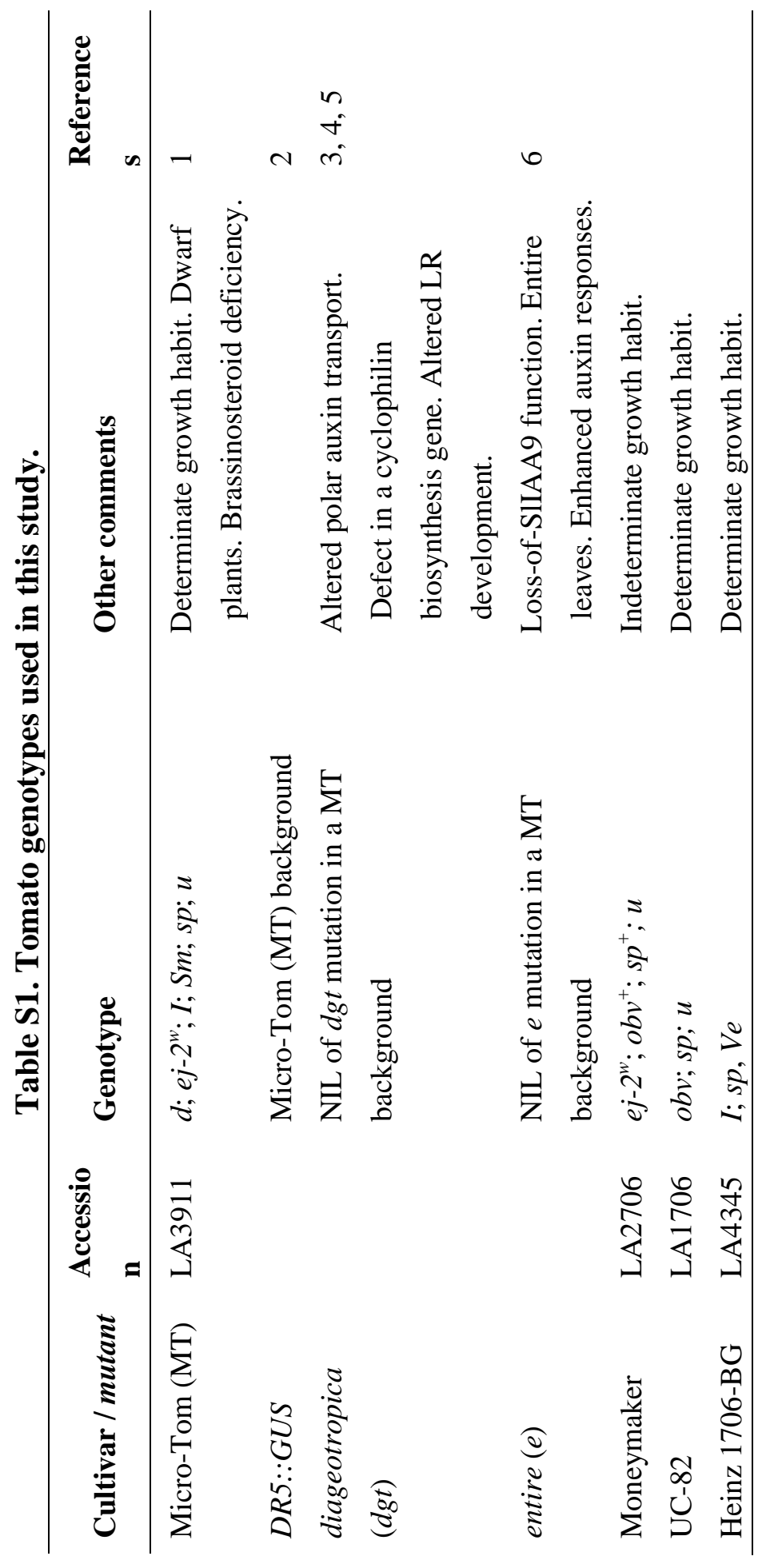




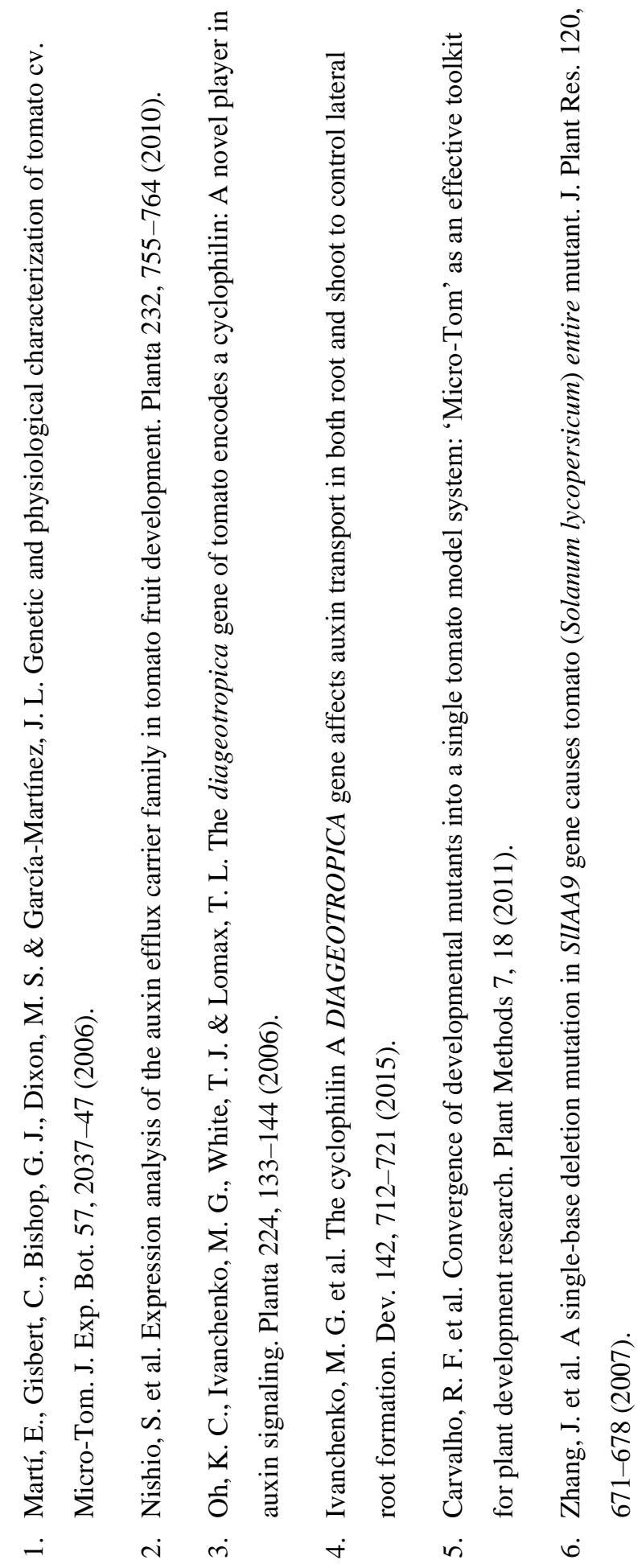




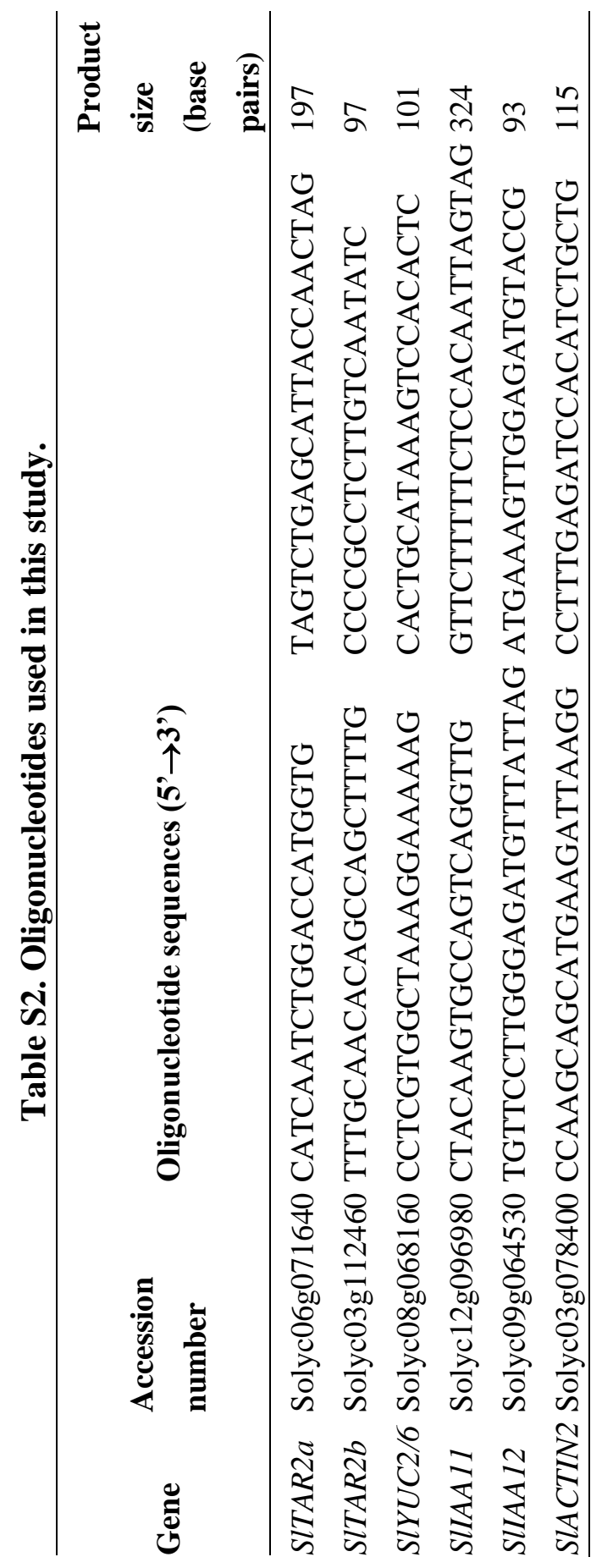

